\title{
Stormtime particle energization with high temporal resolution AMIE potentials
}

\author{
George V. Khazanov, ${ }^{1}$ Michael W. Liemohn, ${ }^{2}$ Mei-Ching Fok, ${ }^{3}$ Timothy S. Newman, ${ }^{4}$ \\ and Aaron J. Ridley ${ }^{2}$
}

Received 6 August 2003; revised 26 November 2003; accepted 14 January 2004; published 13 May 2004.

[1] Simulations were conducted to investigate the influence of rapid electric field fluctuations on electron energization in the inner magnetosphere based on the assimilative mapping of ionospheric electrodynamics (AMIE) technique. Simulations for four different magnetic storms were run, namely those that occurred on 15 May 1997, 4 May 1998, 25 September 1998, and 19 October 1998. Here we have examined the formation of highenergy $(100-500 \mathrm{keV})$ electrons in the inner magnetosphere during these storm events with our recently developed relativistic radiation belt transport code. The point of this numerical experiment is to show that a simulation of a real event must have the high time resolution electric field input files in order to produce the seed population for the radiation belts, which are often observed to increase in the days following a magnetic storm.

Specifically, a cadence of the global electric field pattern of $5 \mathrm{~min}$ or less produces inner magnetospheric fluxes that are larger (by up to several orders of magnitude) than fluxes produced with a longer cadence. Differences were particularly large relative to simulation results with a 3-hour time cadence, analogous to a Kp-driven electric field

model. INDEX TERMS: 7807 Space Plasma Physics: Charged particle motion and acceleration; 2712 Magnetospheric Physics: Electric fields (2411); 2788 Magnetospheric Physics: Storms and substorms; 2720 Magnetospheric Physics: Energetic particles, trapped; KEYWORDS: ring current, radiation belts, magnetic storms, particle acceleration

Citation: Khazanov, G. V., M. W. Liemohn, M.-C. Fok, T. S. Newman, and A. J. Ridley (2004), Stormtime particle energization with high temporal resolution AMIE potentials, J. Geophys. Res., 109, A05209, doi:10.1029/2003JA010186.

\section{Introduction}

[2] One of the largest uncertainties associated with modeling the injection of the ring current is the description of the large-scale electric field. Created by a day-night pressure difference in the magnetic flux in the magnetosphere (from an imbalance in reconnection rates), this field convects charged particles from the magnetotail into the inner magnetosphere. Its dynamical configuration near the Earth is still a subject of debate.

[3] Until recently, our understanding of the inner magnetospheric electric field was limited to our interpretation of in situ measurements. Several empirical electric field models were created, such as those by Nishida [1966] and Brice [1967], Volland [1973] and Stern [1975], and McIlwain [1974, 1986]. Satellites such as CRRES have also probed the inner magnetospheric electric field, yielding statistical

\footnotetext{
${ }^{1}$ National Space Science and Technology Center, NASA Marshall Space Flight Center, Huntsville, Alabama, USA.

${ }^{2}$ Space Physics Research Laboratory, University of Michigan, Ann Arbor, Michigan, USA.

${ }^{3}$ Laboratory for Extraterrestrial Physics, NASA Goddard Space Flight Center, Greenbelt, Maryland, USA.

${ }^{4}$ Computer Science Department, University of Alabama in Huntsville, Huntsville, Alabama, USA.

Copyright 2004 by the American Geophysical Union. 0148-0227/04/2003JA010186\$09.00
}

compilations counter to the empirical models [Rowland and Wygant, 1998]. That is, rather than being dominated by the shielding effect with little or no electric field near the Earth, the fields are highly variable (up to $6 \mathrm{mV} / \mathrm{m}$ ) in the nearEarth nightside (at $2<\mathrm{L}<6$ ) for large $\mathrm{Kp}$ [Wygant et al., 1998]. Ionospheric observations such as those made by the DMSP satellite [e.g., Burke et al., 1998; Anderson et al., 2001] and ground-based radars [e.g., Foster et al., 1986; Fejer et al., 1990; Yeh et al., 1991, Foster and Vo, 2002] also show high variability in the subauroral nightside during active times. Only recently (from the IMAGE satellite) have global snapshots of the plasma morphology in the inner magnetosphere been obtained with a quality suitable for detailed, quantitative analysis [Burch et al., 2001], and progress in extracting the electric field from these images is slow [e.g., Goldstein et al., 2003].

[4] Because the field lines of the inner magnetosphere pass through the midlatitude ionosphere, the convection patterns here are often useful to help explain the dynamics at the higher altitudes. For instance, Jordanova et al. [2001] showed that the statistical model of Weimer [1996] produces better comparisons of simulated ion flux against in situ data than do results using the classic Volland-Stern electric field model. Another ionospheric convection description is the assimilative mapping of ionospheric electrodynamics (AMIE) technique [Richmond and Kamide, 1988]. This approach uses any available 
ionospheric data for a given time to produce an instantaneous convection pattern. Boonsiriseth et al. [2001] discussed a semiempirical method of mapping these convection patterns out from the ionosphere into the inner magnetosphere, finding that the resulting field was more realistic than the Volland-Stern field. Chen et al. [2003] showed that the AMIE-derived equipotentials concentrate in the evening sector during the main phases of storms, moving plasma sheet ions into $\mathrm{L} \sim 3-4$ (even $\mathrm{L} \sim 2$ for extremely large events) in less than $30 \mathrm{~min}$.

[5] In a study by Khazanov et al. [2004], several electric field models were used in conjunction with a ring current electron and ion transport model to examine the differences in the net acceleration to the particles. It was shown that narrow channels of high electric field are an effective mechanism for injecting plasma into the inner magnetosphere. Analytical expressions for the electric field cannot produce these channels of intense plasma flow and thus result in less entry and energization of the plasma sheet into near-Earth space. For the ions, omission of these channels leads to an underprediction of the strength of the stormtime ring current and therefore an underestimation of the geoeffectiveness of the storm event. For the electrons, omission of these channels leads to the inability to create a seed population deep in the inner magnetosphere. These electrons can eventually be accelerated into $\mathrm{MeV}$ radiation belt particles due to the different type of acceleration mechanisms [e.g., Li et al., 1993; Hudson et al., 1996; Summers and Ma, 2000; Albert, 2001; Fok et al., 2001].

[6] In this study, simulations are conducted to investigate the influence of rapid electric field fluctuations on electron energization in the inner magnetosphere based on the AMIE technique. Simulations for four different magnetic storms were run, namely those that occurred on 15 May 1997, 4 May 1998, 25 September 1998, and 19 October 1998. Specifically, we examine the formation of high-energy (10$1000 \mathrm{keV}$ ) electrons in the inner magnetosphere during these storm events with our relativistic radiation belt transport code that we have been developing over the past year [Khazanov et al., 2004].

\section{Model Description}

[7] We simulate the magnetospheric dynamics of hot electrons by solving the bounce-averaged kinetic equation for the phase space distribution function, $Q_{e}$

$$
\begin{aligned}
\frac{\partial Q_{e}}{\partial t} & +\frac{1}{R_{o}^{2}} \frac{\partial}{\partial R_{o}}\left(R_{o}^{2}\left\langle\frac{d R_{o}}{d t}\right\rangle Q_{e}\right)+\frac{\partial}{\partial \varphi}\left(\left\langle\frac{d \varphi}{d t}\right\rangle Q_{e}\right) \\
& +\frac{1}{\gamma \sqrt{\gamma^{2}-1}} \frac{\partial}{\partial E}\left(\gamma \sqrt{\gamma^{2}-1}\left\langle\frac{d E}{d t}\right\rangle Q_{e}\right) \\
& +\frac{1}{f\left(\mu_{o}\right) \mu_{o}} \frac{\partial}{\partial \mu_{o}}\left(f\left(\mu_{o}\right) \mu_{o}\left\langle\frac{d \mu_{o}}{d t}\right\rangle Q_{e}\right)=-\frac{Q_{e}}{\tau_{a t m}}-\frac{Q_{e}}{\tau_{w}}+\frac{\delta Q_{e}}{\delta t}
\end{aligned}
$$

as a function of position in the magnetic equatorial plane $\left(R_{0}, \varphi\right)$, kinetic energy and the cosine of the equatorial pitch angle $\left(E, \mu_{0}\right)$, and time $t$. Using an arbitrary convection model and a dipole magnetic field, the bounce-averaged velocity terms in equation (1) from adiabatic drift can be written in the form [Khazanov et al., 1999]

$$
\begin{aligned}
& \left\langle\frac{d R_{0}}{d t}\right\rangle=-\frac{R_{0}^{2}}{M_{E}} \frac{\partial \Phi}{\partial \varphi} \\
& \left\langle\frac{d \varphi}{d t}\right\rangle=\frac{C}{M_{E}}+\frac{R_{0}^{3}}{M_{E}} \frac{\partial \Phi}{\partial R_{0}}-\frac{3 m c^{2} R_{0}}{2 e M_{E}} \frac{\left(\gamma^{2}-1\right)}{\gamma}\left[1-\frac{I\left(\mu_{0}\right)}{6 h\left(\mu_{0}\right)}\right] ; \\
& \left\langle\frac{d E}{d t}\right\rangle=-\frac{3 m c^{2}}{2 R_{0}} \frac{\gamma^{2}-1}{\gamma}\left[1-\frac{I\left(\mu_{0}\right)}{6 h\left(\mu_{0}\right)}\right]\left\langle\frac{d R_{0}}{d t}\right\rangle ; \\
& \left\langle\frac{d \mu_{0}}{d t}\right\rangle=\frac{\left(1-\mu_{0}^{2}\right)}{4 R_{0} \mu_{0}} \frac{I\left(\mu_{0}\right)}{h\left(\mu_{0}\right)}\left\langle\frac{d R_{0}}{d t}\right\rangle \text {; } \\
& I\left(\mu_{0}\right)=\frac{1}{R_{0}} \int^{s_{m \prime}} \sqrt{1-B(s) / B_{m}} d s
\end{aligned}
$$

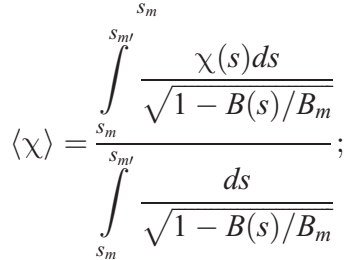

$$
\begin{aligned}
& \tau_{a t m}=\left\{\begin{array}{ll}
\frac{2 R_{0} \gamma h}{c \sqrt{\gamma^{2}-1}} & \alpha_{0}<\alpha_{0 L C} \\
\infty & \alpha_{0}>\alpha_{0 L C}
\end{array} ;\right. \\
& \mu_{0}=\cos \alpha_{0} \quad h\left(\mu_{0}\right)=\frac{1}{2 R_{0}} \int_{s_{m}}^{s_{m \prime}} \frac{d s}{\sqrt{1-B(s) / B_{m}}},
\end{aligned}
$$

where $\phi$ is the electric potential distribution, $\alpha_{0, \mathrm{LC}}$ is the loss cone boundary, subscripts $m, m^{\prime}$ mark the conjugate mirror points on the field line, and $M_{E}$ represents the Earth dipole moment.

[8] Note that the convection drift is written as a derivative of an arbitrary magnetospheric electric potential $\Phi$. If one assumes that the electric field is homogeneous and directed from dawn to dusk in the equatorial plane, then we have

$$
\frac{\partial \Phi}{\partial \varphi}=A R_{0} \cos \varphi \quad \text { and } \quad \frac{\partial \Phi}{\partial R_{0}}=A \sin \varphi
$$

where $\mathrm{A}$ is an activity-dependent strength parameter of units $\mathrm{V} \mathrm{m}^{-1}$ [Volland, 1973; Stern, 1975]. If one assumes that the electric potential is like that used by Liemohn et al. [2001], then the convection drift terms reduce to those given in equation (11) of that paper.

[9] The bounce-averaged distribution function in equation (1), $Q\left(t, R_{0}, \varphi, \mu_{0}, E\right)$, is associated with the number of particles with kinetic energy and cosine of equatorial pitch angle between $E+$ and $E+d E$, and $\mu_{0}$ and $\mu_{0}+d \mu_{0}$, whose gyrocenters are distributed on a given magnetic field line on the interval $s_{m}<s<s_{m^{\prime}}$ at time $t$ as

$$
d n=8 \pi \mathrm{cm}^{2} h \gamma \sqrt{\gamma^{2}-1} Q d E \mu_{0} d \mu_{0} d \varphi R_{0}^{2} d R_{0}
$$




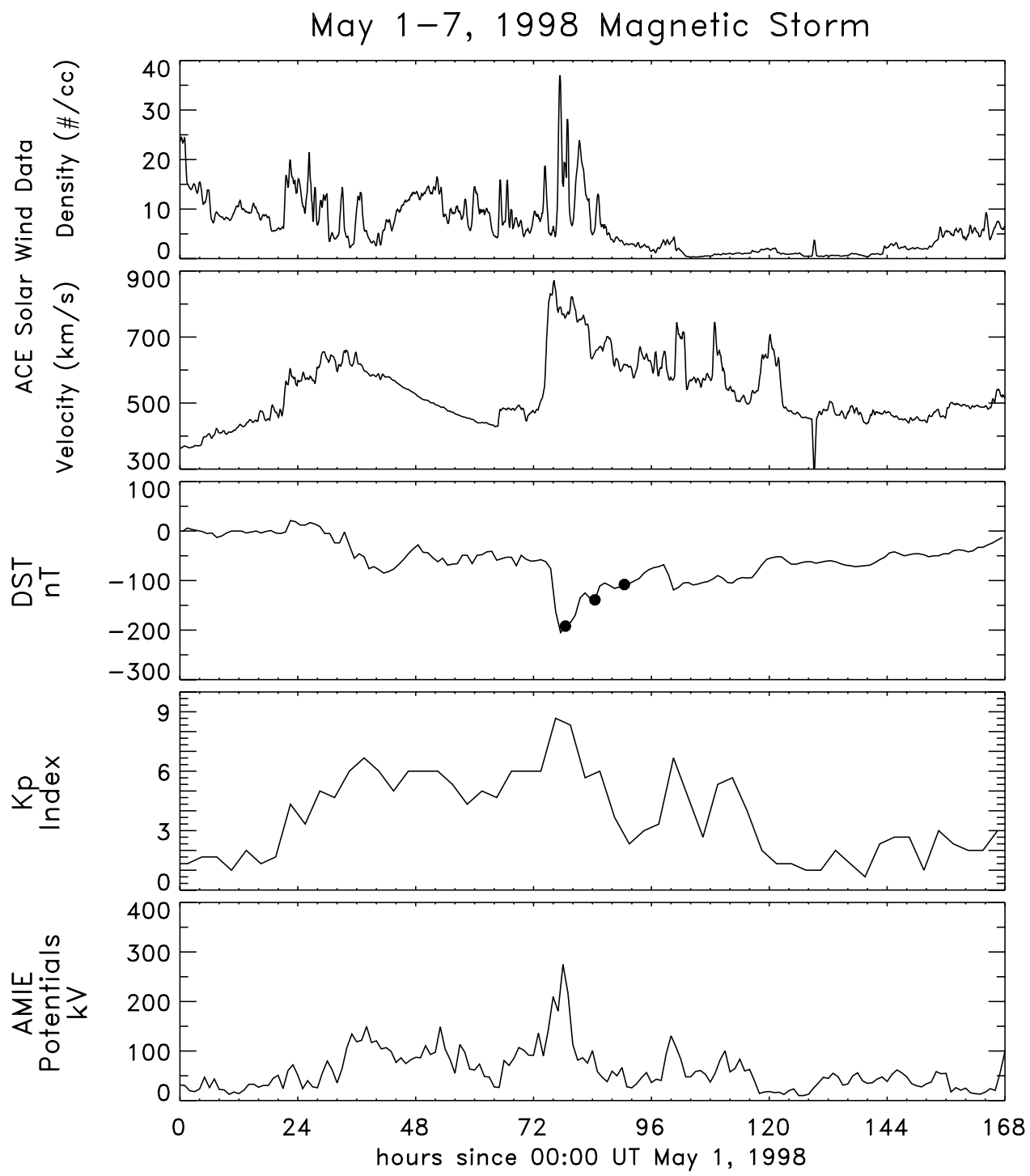

Figure 1. Geophysical quantities during the 1-7 May 1998 magnetic storm. The first two rows are the solar wind density and velocity. The next two rows show the Dst and Kp global indices. The final panel shows the total cross-tail potential differences during the 1-7 May 1998 storm from the AMIE model. The black dots on the Dst time series are the three times highlighted for detailed analysis in Figures 2, 3, and 4.

Note that equation (1) is written in relativistic form, where

$$
\gamma=1+\frac{E}{m c^{2}}
$$

and all of the coefficients in equation (1) are converted accordingly. All of the bounce-averaged quantities in equation (1) are denoted with $\langle\ldots\rangle$. On the left-hand side, the bracketed terms are the drifts speeds in configuration and velocity speed that include all electric and magnetic drifts. These velocities, one for each independent variable in the calculation, will be determined from a number of electric field descriptions, to be discussed below. The $f\left(\mu_{0}\right)$ coefficient is a bounce-averaging term that ranges from 0.74 to 1.38 [Ejiri, 1978]. On the right-hand side, the first term represents precipitation of particles into the atmosphere (assumed lost at $800 \mathrm{~km}$ altitude along each field line). For the electrons, pitch angle diffusion into the loss cone resulting from interactions with whistler mode waves in the plasmasphere is taken into account via the attenuation factors $\tau_{w}$ in equation (1) based on the Lyons et al. [1972] study. The last term on the right-hand side of equation (1) includes losses from Coulomb collisions (pitch angle scattering and energy degradation terms). The energy range of the simulations extends from $10 \mathrm{eV}$ to $5 \mathrm{MeV}$. For further details about the numerical solution of the kinetic equation (1), please see Jordanova et al. [1996, 1997], Khazanov et al. [1998, 1999], and Liemohn et al. [2001].

[10] It should be stressed that the classical radial diffusion process [Schulz and Lanzerotti, 1974] is omitted from equation (1). The diffusion process is a fairly slow one and does not explain the rapid ( $\sim$ hours) rise in the electron flux. Instead, we take into account electric field fluctuations on a timescale comparable with or less than the drift period 

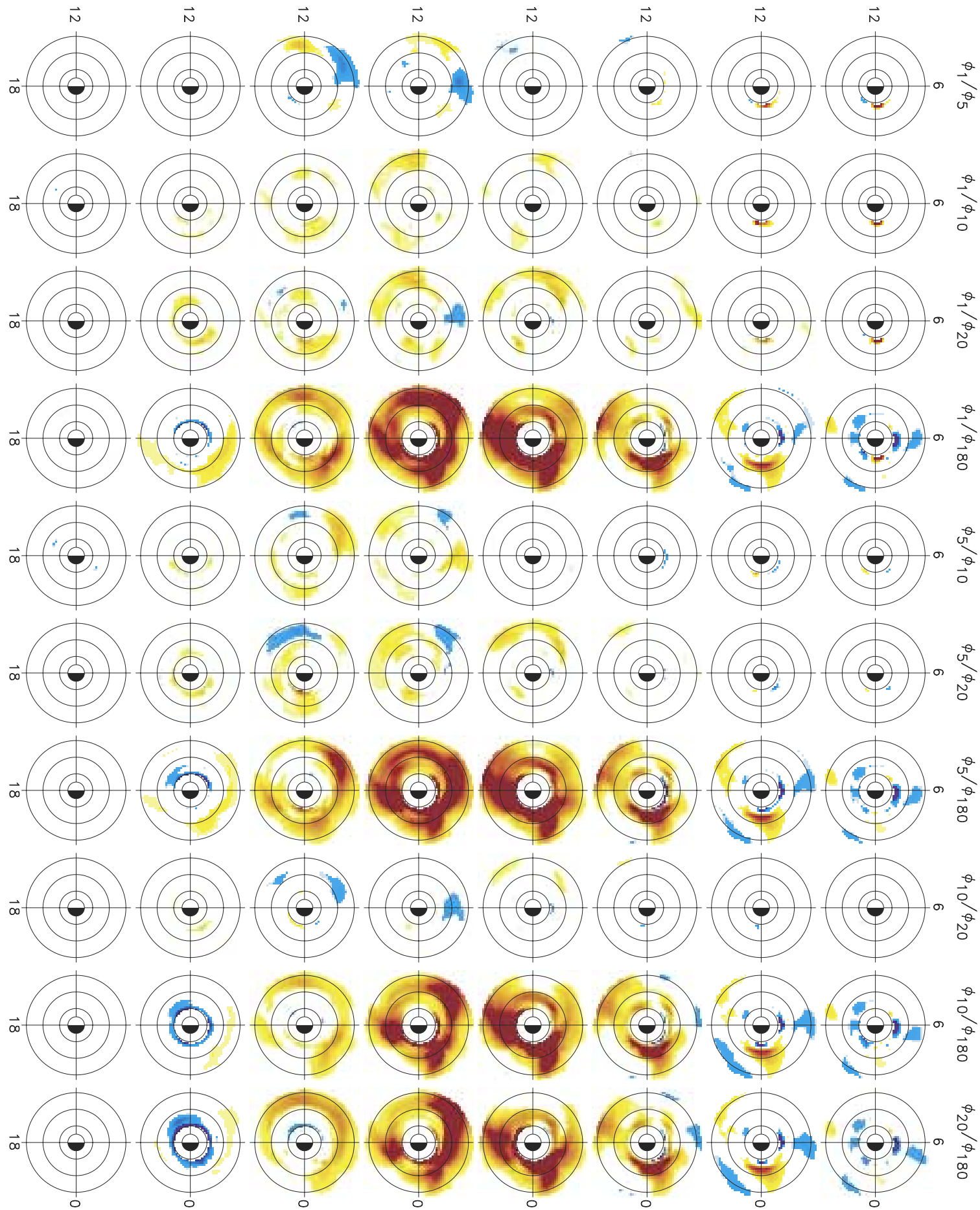

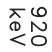

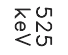

주에

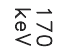

중

$\stackrel{\pi}{i} \in$
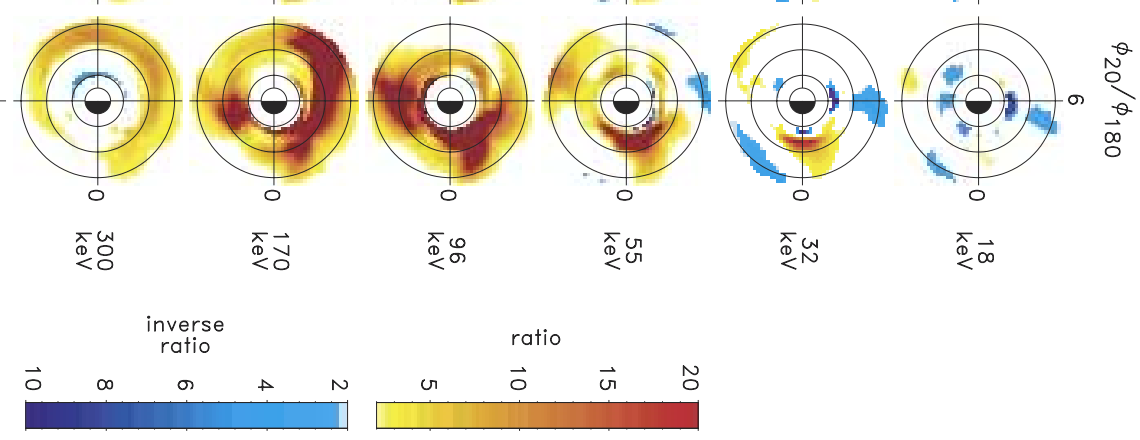

Figure 2. Electron flux ratios between simulations with the E-field update time cadences given by the subscripts (as defined in the text) at hour 78 of the simulation (0600 UT on 4 May 1998). Each row is a different energy channel, as indicated. Colors in the yellow-red range indicate ratios where the shortertime-cadence simulation is larger than the longer-time-cadence simulation flux by at least a factor of two. Colors in the blue-purple range indicate that the longer-time-cadence simulation flux is at least twice as large as the shorter-time-cadence simulation flux. In each plot, the view is over the north pole, with the Sun to the left and thin black circles drawn at $\mathrm{L}=2,4$, and 6 . 

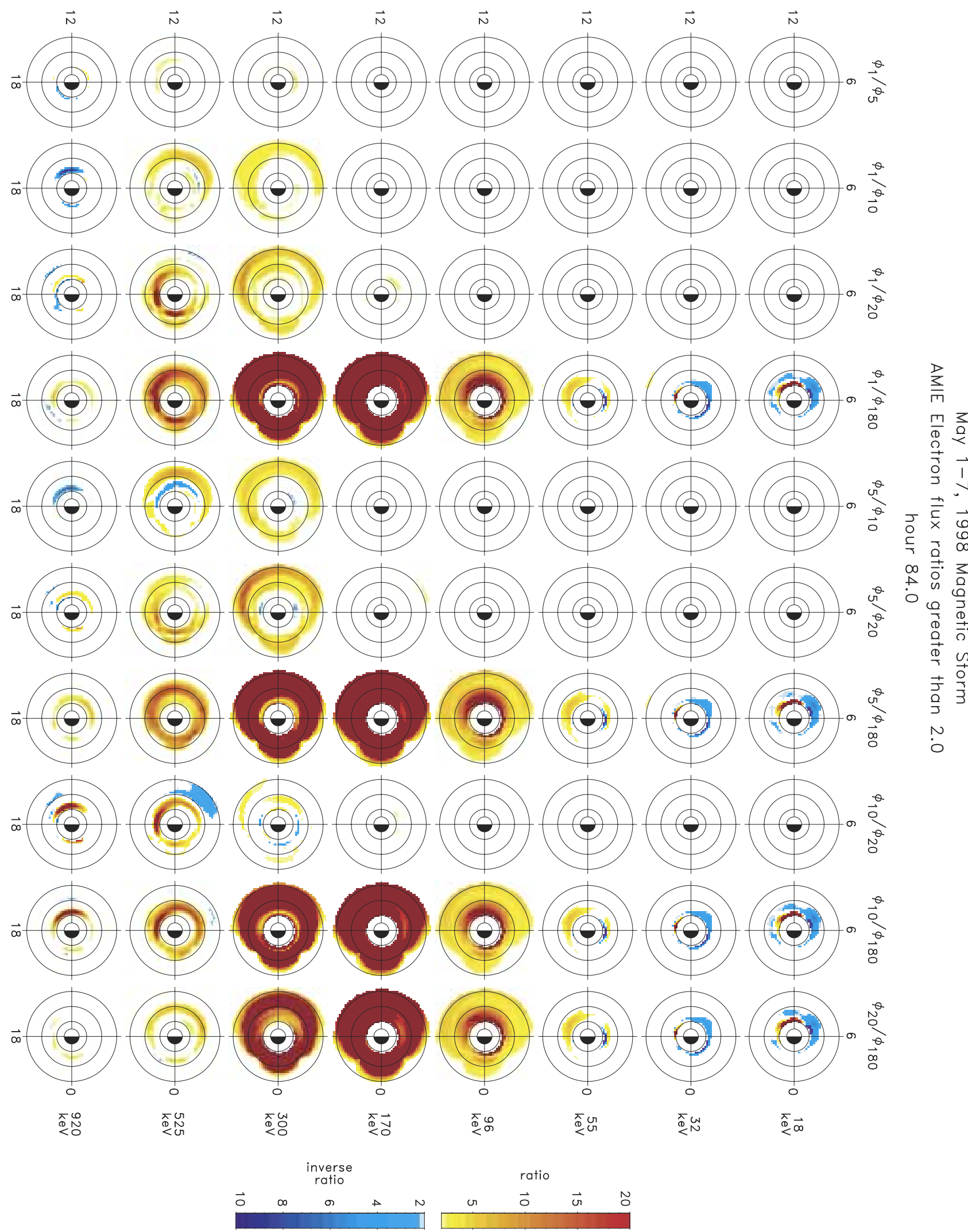

Figure 3. Same as Figure 2 except that the ratios are for hour 84 of the simulation (1200 UT on 4 May 1998). 

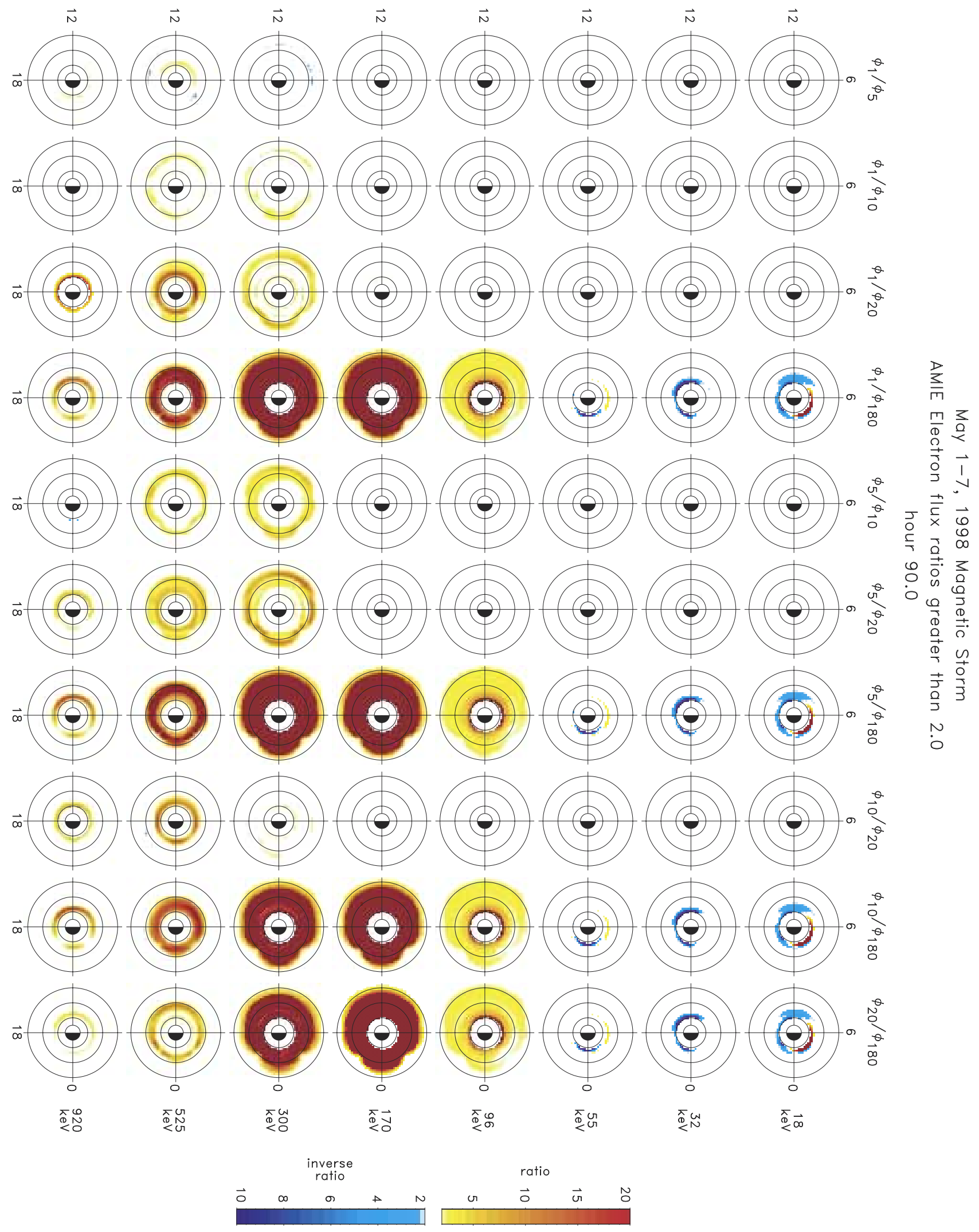

Figure 4. Same as Figure 2 except that the ratios are for hour 90 of the simulation (1800 UT on 4 May 1998). 

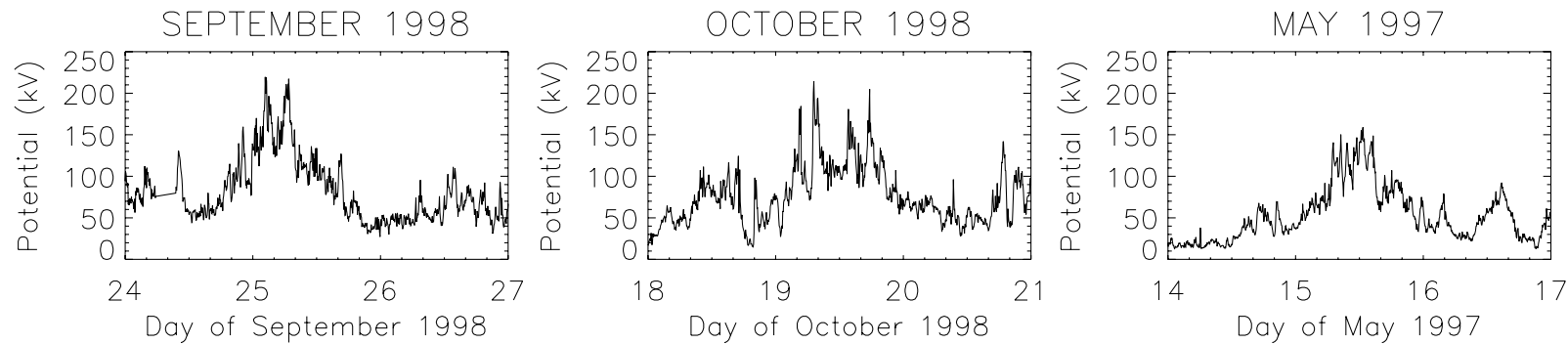

Figure 5. Total cross polar cap potential difference as calculated by the AMIE routine for the three storms of (left) 25 September 1998, (middle) 19 October 1998, and (right) 15 May 1997.

of radiation belt electrons from the realistic potential distributions provided by the AMIE model. Radial diffusion by nature is a stochastic process that is driven by electric and magnetic field fluctuations that are included in the model through electric and magnetic drifts.

\section{The 1-7 May 1998 Storm Results}

[11] To investigate the influence of rapid electric field fluctuations on electron energization in the inner magnetosphere, we have selected the 1-7 May 1998 storm and used five different time resolutions of the AMIE electric field model. The interplanetary coronal mass ejection (ICME) that hit the magnetosphere on 4 May 1998 was a large magnetic cloud with a trailing high-speed stream, and details of the solar wind and interplanetary magnetic field can be found in numerous studies [e.g., Gloeckler et al., 1999; Skoug et al., 1999; Farrugia et al., 2002]. It is actually the high-speed stream that hits early on 4 May 1998, after the cloud had passed by the Earth on 2-3 May 1998. Figure 1 shows an overview of the geophysical response to this activity during the 1-7 May 1998 interval. Figures $1 \mathrm{a}$ and $1 \mathrm{~b}$ show the solar wind density and velocity, respectively, from the ACE spacecraft. The Dst index shown in Figure 1c is an hourly value of the globally averaged midlatitude magnetic perturbation in the northsouth direction. In Figure $1 \mathrm{~d}, \mathrm{Kp}$ is a 3-hour cadence global activity index related to the amount of fluctuation in auroralzone groundbased magnetometer records. Figure 1e shows the total cross-tail potential difference for the AMIE model. It is seen that the AMIE potential difference spikes up to around $250 \mathrm{kV}$ during the main phase injection early on 4 May 1998.

[12] All of the electron transport code simulations use kappa distributions applied at the outer simulation boundary (at 6.6 $R_{E}$ equatorial distance) for the source populations of electrons [e.g., Ma and Summers, 1999]

$j(\mathbf{r}, E)=n(\mathbf{r}) \frac{\Gamma(\kappa+1)}{(\pi \kappa)^{3 / 2} \Gamma(\kappa-1 / 2)}\left(\frac{1}{2 m_{\mathrm{o}} E_{\mathrm{o}}}\right)^{1 / 2} \frac{E}{E_{\mathrm{o}}}\left(1+\frac{E}{\kappa E_{\mathrm{o}}}\right)^{-\kappa-1}$,

where the differential number flux $j$ is related to the distribution function by $j=2 E Q / \mathrm{m}^{2}$. In order to isolate the effects of the electric field configuration, all five simulations are run with exactly the same initial and boundary conditions for the inner magnetospheric plasma populations.
Equation (2) is used with $\kappa=3$ and the parameters $n$ and $E_{0}$ specified by the upstream solar wind

$$
\begin{aligned}
& n(t)\left[\mathrm{cm}^{-3}\right]=0.025 N_{s w}(t-3 h)+0.395 \\
& E_{0}(t)[\mathrm{keV}]=0.02 V_{s w}(t-3 h)-3.0
\end{aligned}
$$

as determined from data [Borovsky et al., 1998; Ebihara and Ejiri, 2000]. The variations of these boundary condition quantities follow the solar wind density and velocity time series as shown in Figures 1a and 1b, respectively. We use the results of the NASA AE- 8 model as the initial condition for each run.

[13] The only difference between the numerical experiments was in the convection electric field description. They all use electric potential values from the AMIE technique, which ingests ground-based and satellite measurements to obtain a best-fit potential pattern for a given instant but with different time resolutions. The first run used AMIE patterns with a 1-min cadence. The magnetometer data, which dominates the AMIE assimilation (especially at subauroral latitudes where there is no radar data), has a 1-min cadence. The radar data (2-min resolution) and satellite data ( $\sim 20 \mathrm{~min}$ per polar passage) are weighted in time to spread their influence over the window of observation. Furthermore, Ridley et al. [1998, 1999] discussed the validity of this time resolution of AMIE potential patterns, finding that 1-min cadence patterns contain real magnetospheric variations. The second through fifth runs used AMIE patterns with cadences of $5,10,20$, and $180 \mathrm{~min}$, respectively. The electric field patterns for runs 2 through 5 were obtained simply by taking an average of the 1 -min values. For each time step in the simulation (which are less than $1 \mathrm{~min}$, typically $5-10 \mathrm{~s}$ ), the potential pattern for that moment is interpolated between the closest two patterns. Therefore while the electric potential pattern (and therefore the convection electric field) is changing every time step, the global dynamics of the pattern are shifting on 1-min, 5-min, 10-min, 20-min, or 3-hour timescales for the five simulations, respectively.

[14] Results from this experiment are shown in Figures 2 through 4. Each of these figures shows the result at a given universal time during the storm event. Hour 78 (Figure 2) is 0600 UT on 4 May 1998, hour 84 (Figure 3) is 1200 UT on 4 May 1998, and hour 90 (Figure 4) is 1800 UT on 4 May 1998. Each subplot is a view of the inner magnetosphere from over the North Pole, so noon is to the left and dawn is to the top. Each column of each figure is a comparison of 

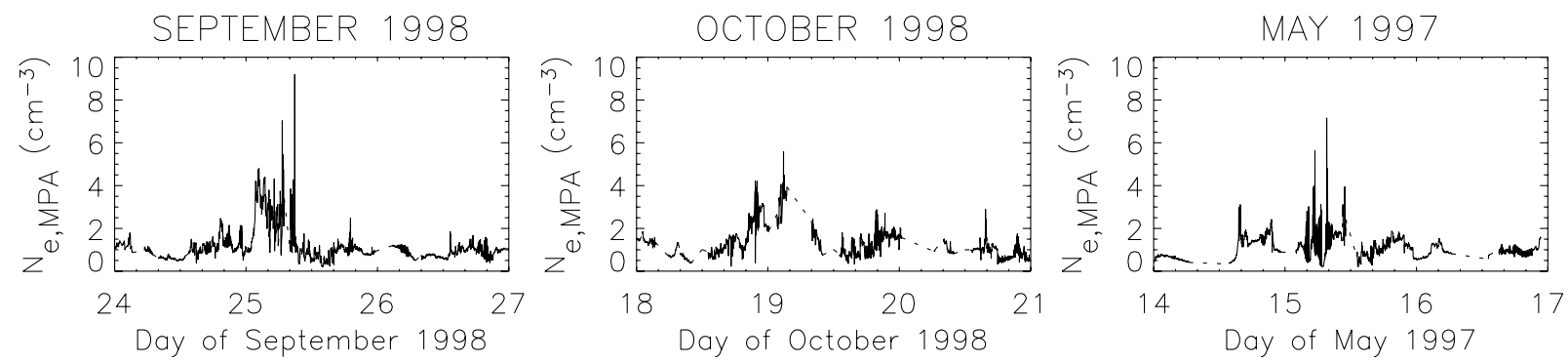

Figure 6. Nightside electron density compiled from measurements from the MPA instrument on the LANL satellites for the three storms of (left) 25 September 1998, (middle) 19 October 1998, and (right) 15 May 1997.

the fluxes for two of the simulations, designated by subscripts " 1 ," " 5 ," “ 10 ," " 20 ," and " 180 ," for the respectively higher time-cadence E-field inputs. The eight rows show results at eight energies, the numerical grid cells nearest to $20 \mathrm{keV}, 30 \mathrm{keV}, 55 \mathrm{keV}, 100 \mathrm{keV}, 170 \mathrm{keV}$, $300 \mathrm{keV}, 525 \mathrm{keV}$, and $920 \mathrm{keV}$, respectively. A flux ratio above +2.0 is colored yellow-red when the flux of the shorter time cadence E-field update simulation is larger than the flux of the simulation with the longer time cadence between E-field updates. A value below-2.0 is colored bluepurple when the flux of the longer time cadence run is larger than the flux of the shorter time cadence run. That is, color appears in the dial plots only when one of the simulations produced fluxes at least twice as large as those from the other simulation. Note that the colorscale ranges are slightly different in the two directions (twice as big for positive values). Absolute values of the flux intensities are not shown here because we have previously shown them in earlier studies [e.g., Khazanov et al., 2002, 2004]. In addition the basic structure of the flux intensities is very similar to those shown in the next section. We refer the reader to these other figures for the details of the inner magnetospheric electron energy spectra.

[15] The time chosen for Figure 2 is when the Dst index reached its deepest minimum during the 1-7 May 1998 period (see Figure 1). Figures 3 and 4 represent times during the recovery phase of the storm, when injection of fresh electrons from the plasma sheet has essentially ceased. Therefore differences in the flux levels between the simulations are due to electric field differences in the inner magnetosphere and not particularly from the injection of new material through the outer boundary of the simulation domain.

[16] The plots become dominated by reds and yellows as time progresses. This means that the electrons are being accelerated through resonant interaction of their drift trajectories with the electric field fluctuations. In the main phase (Figure 2), the largest differences are seen in the 100 and $170 \mathrm{keV}$ energy channels. The increases are localized, often appearing as patches of red throughout the dial plots. The largest increases are seen inside of $\mathrm{L}=4$ on the nightside and beyond $\mathrm{L}=4$ on the dayside. This is due to timing differences in how the plasma sheet electrons are injected and trapped in the inner magnetosphere. The blue ring near $\mathrm{L}=2$ in some of the $500 \mathrm{keV}$ plots is due to the faster E-field updates removing more of the initial condition electrons than the slower E-field updates.

[17] During the recovery phase (Figures 3 and 4), the differences become clearer. For energies less than $100 \mathrm{keV}$, there can be large differences throughout the simulation domain in the data where the AMIE model has been averaged into 3-hour bins (relative to the other simulations). This choice of an averaging timescale is to approximate a Kp-driven simulation, which has a 3-hour time cadence. However, there are often little differences between the 1, 5, 10 , and 20-min update results (the flux ratios have very little color in this case). Going to the high-energy range, the 170 and $300 \mathrm{keV}$ energy channels show the largest differences, especially between the 3-hour cadence results and the shorter time cadence results. Of particularly interest for this study is that the 300 and $500 \mathrm{keV}$ channels show noticeable differences in the flux ratios for all time cadences, except
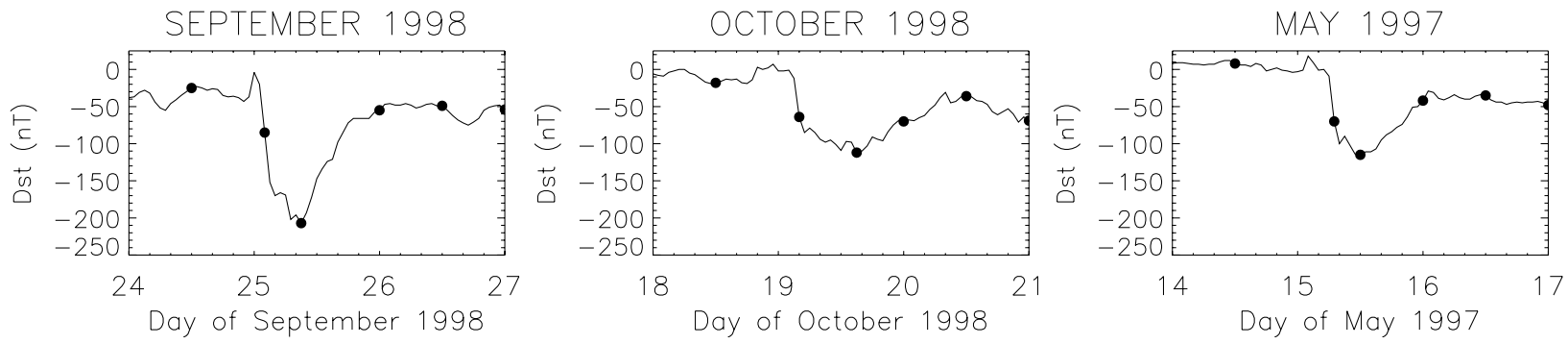

Figure 7. Observed Dst index time series for the three storms of (left) 25 September 1998, (middle) 19 October 1998, and (right) 15 May 1997. The six black dots on each plot are the times chosen for detailed analysis. 

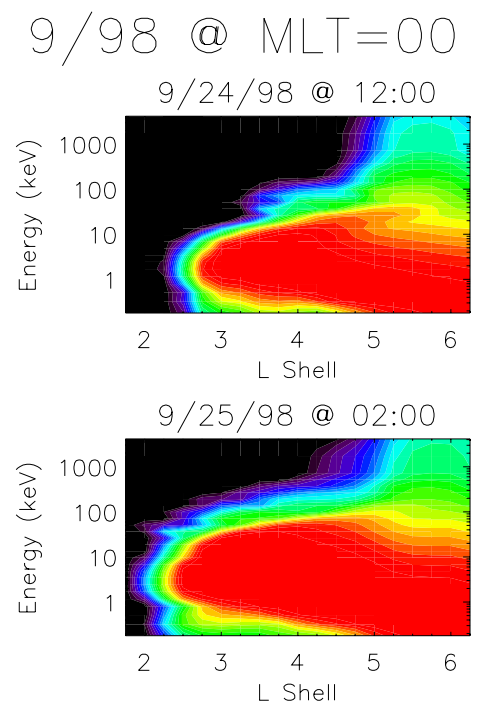

9/25/98@09:00

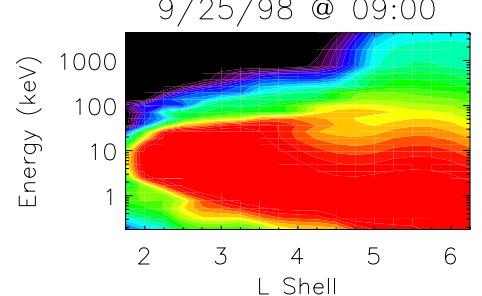

9/26/98@00:00
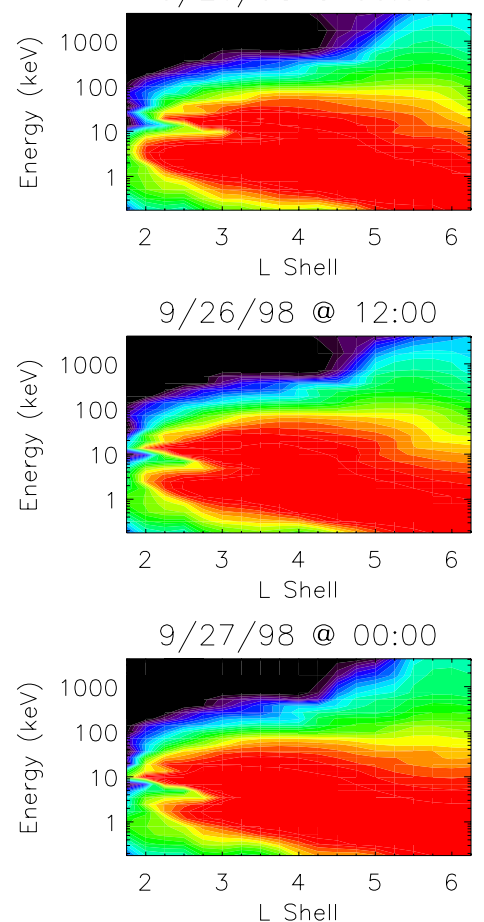

$10 / 98$

@ $M L T=00$

10/18/98@12:00
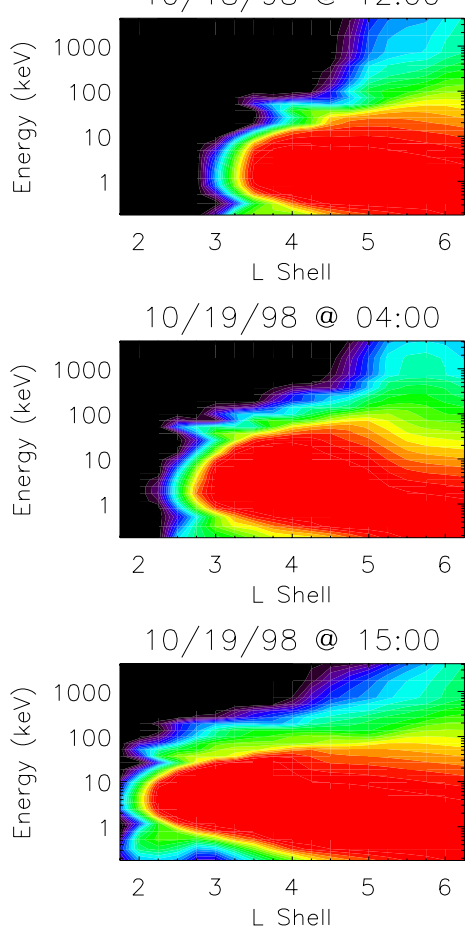

10/20/98@00:00
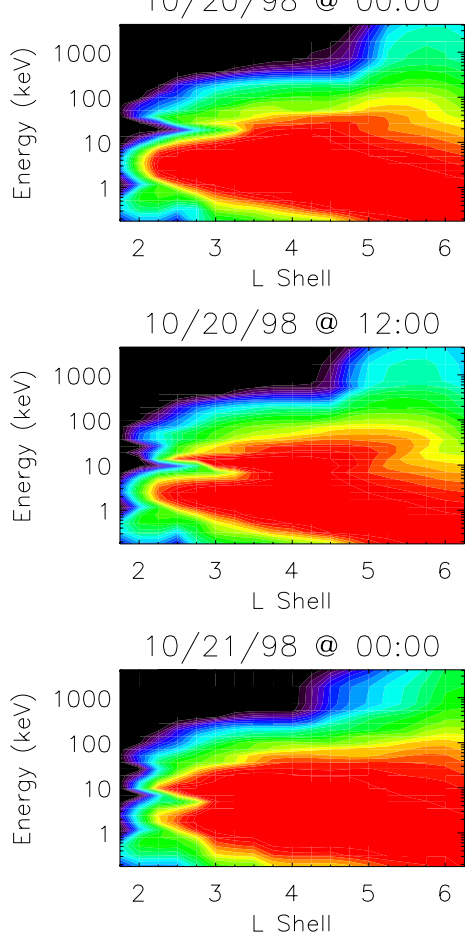

5/97@MLT=00
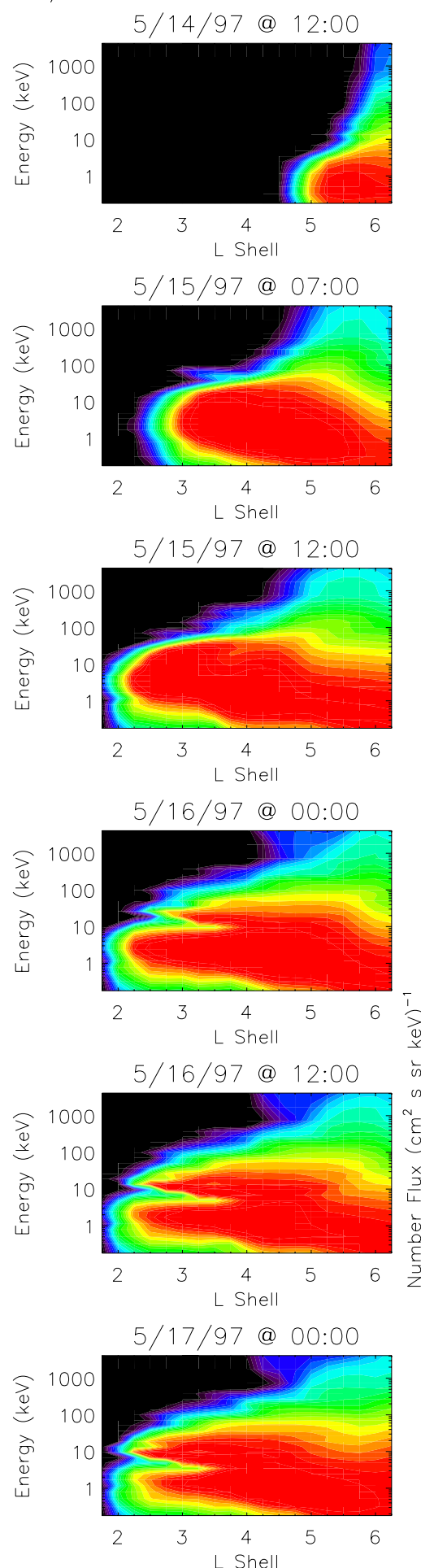

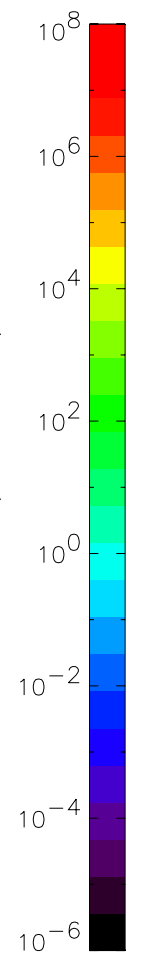

Figure 8. Energy versus L-shell spectrograms of pitch-angle averaged electron flux for the three storms (left column: September 1998, middle column: October 1998, and right column: May 1997). The six rows are at the six times indicated by the black dots in Figure 7. The MLT location for all of these results is local midnight.

between the 1 and 5 -min results. While there is some patchiness to the flux differences in the dial plots at these energies, the main feature is a yellow-to-red ring near $\mathrm{L}=$ $3-5$. This is a critical energy range and spatial location because it is these electrons that are the seed population for the $\mathrm{MeV}$-energy radiation belt electrons produced by localized heating. Little difference is seen between any of the simulations in the highest energy channel $(900 \mathrm{keV})$, 


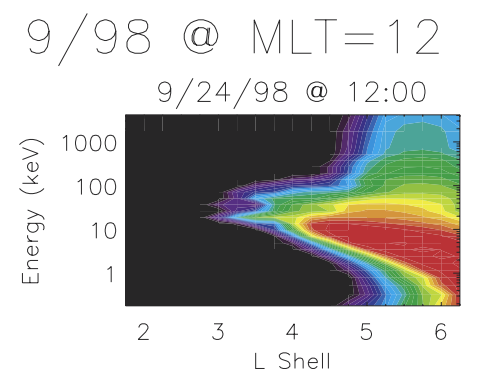

9/25/98@02:00
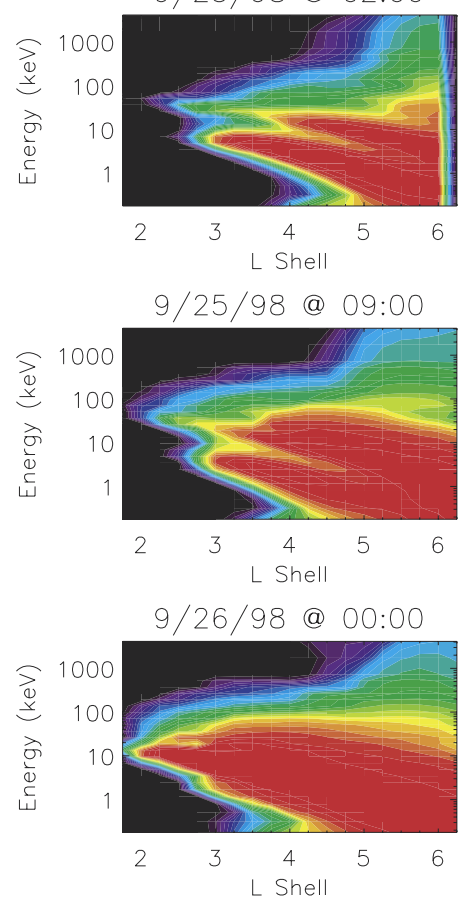

9/26/98@12:00

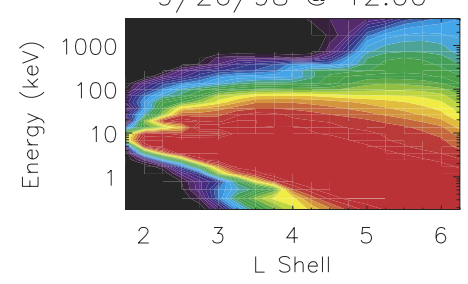

9/27/98@00:00

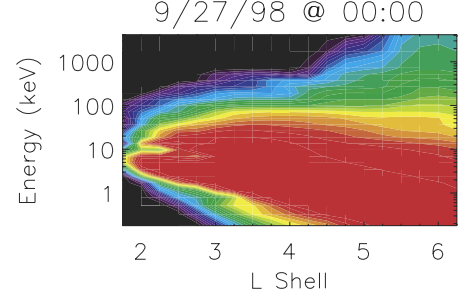

$10 / 98 @ M L T=12$

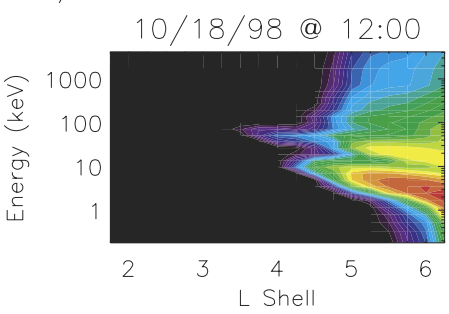

10/19/98@04:00
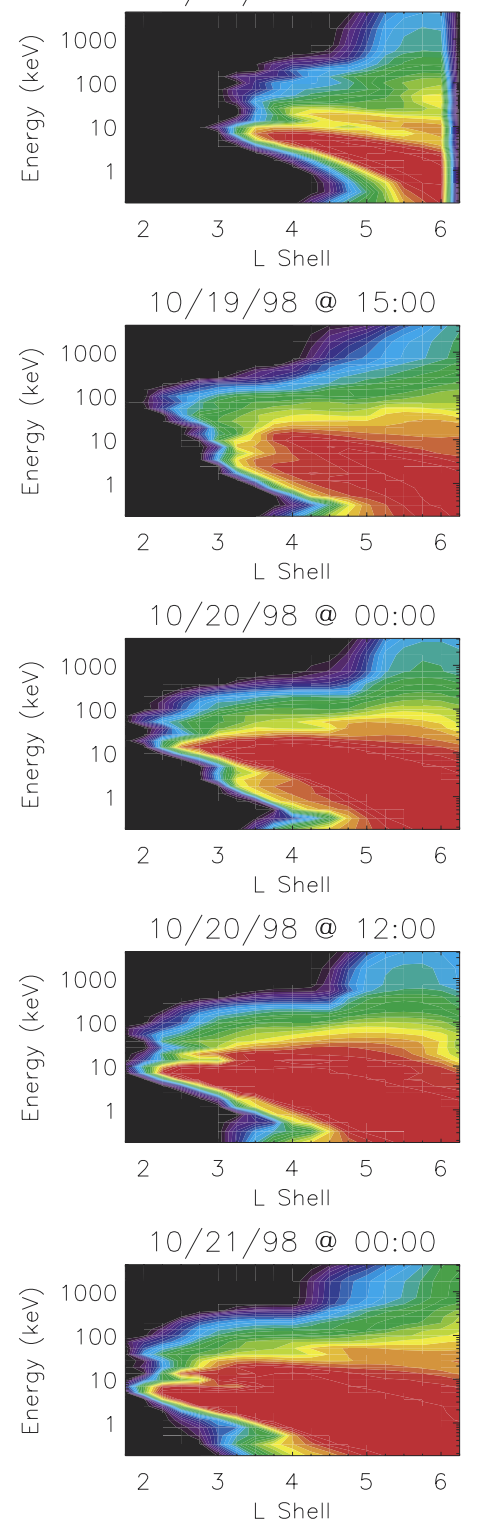
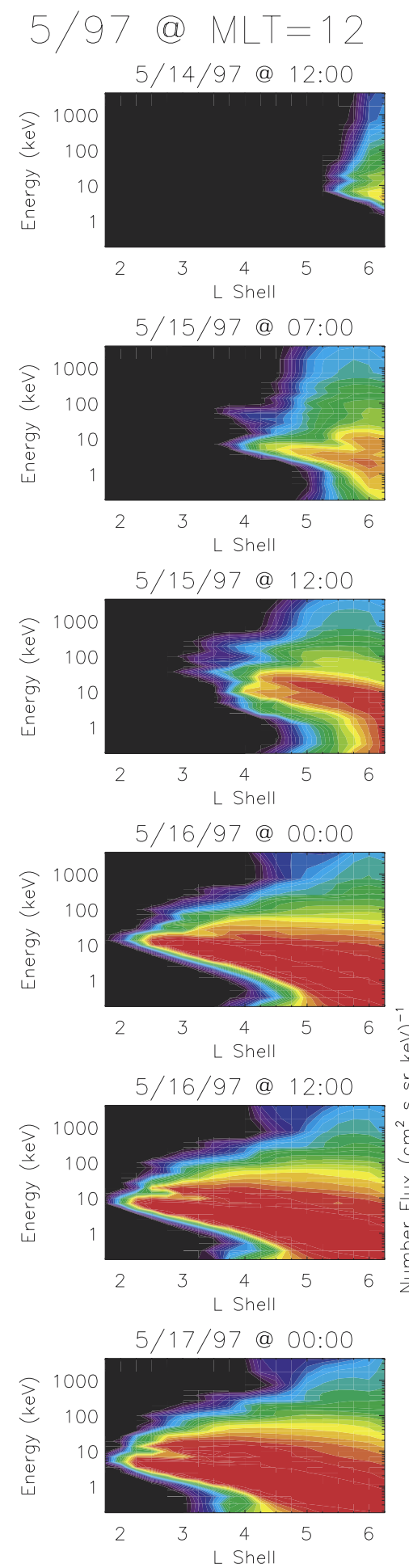

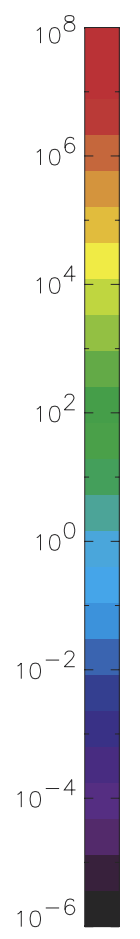

Figure 9. Same as Figure 8, except that the MLT location for all of these results is local noon.

as these particles are relatively unaffected by the electric field.

[18] These results indicate that a 10-min or longer update of the global convection pattern is less efficient at accelerating the electrons than a 1 or 5-min update, which appear to be equally efficient at energization. This suggests that a time cadence for updates of the global electric field pattern of
5 min or less is required to accurately reproduce the real electric field pattern in the inner magnetosphere.

[19] The details of the electron distribution function for the 1-min AMIE potential pattern results were shown in the work of Khazanov et al. [2004]. Here, we are concerned only with the differences between the simulation results with respect to the fluctuations in the global electric field pattern. 


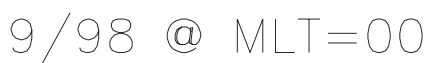

9/24/98@00:00

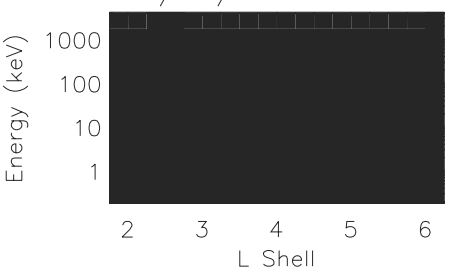

9/24/98@02:00

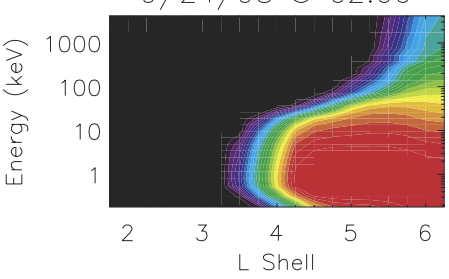

9/24/98@04:00

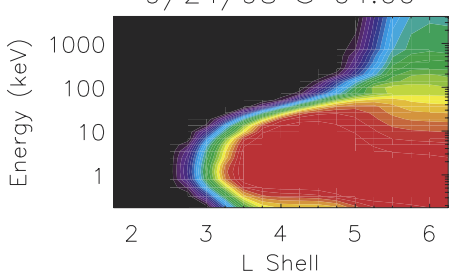

9/24/98@06:00

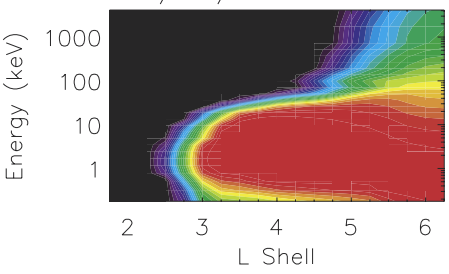

9/24/98@08:00

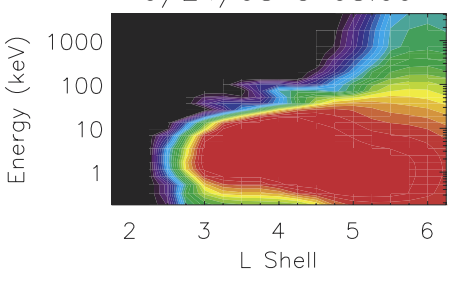

9/24/98@10:00

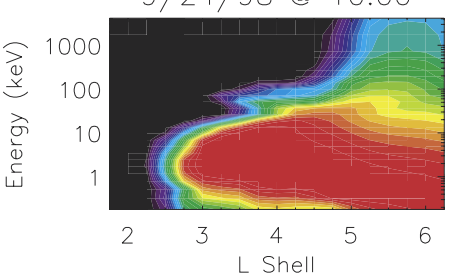

$9 / 98$

@ $M L T=00$

9/24/98@12:00

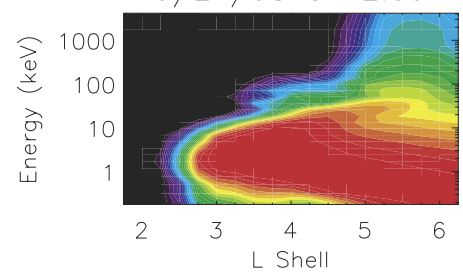

9/24/98@14:00

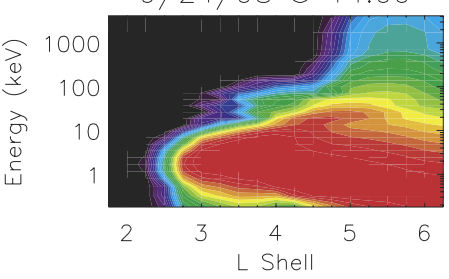

9/24/98@16:00

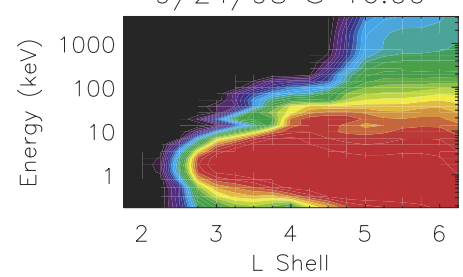

9/24/98@18:00

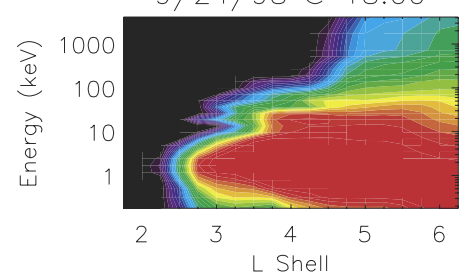

9/24/98@20:00

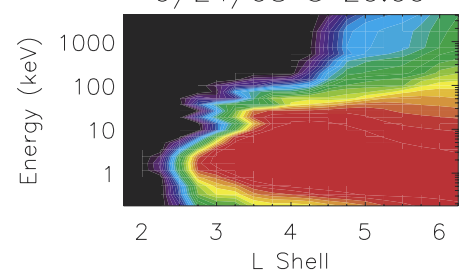

9/24/98@22:00

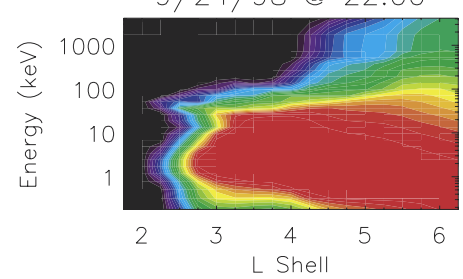

9/98@MLT=00

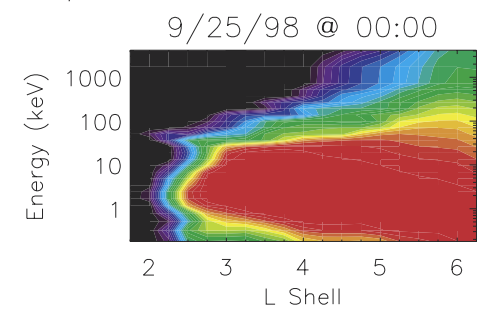

9/25/98@02:00
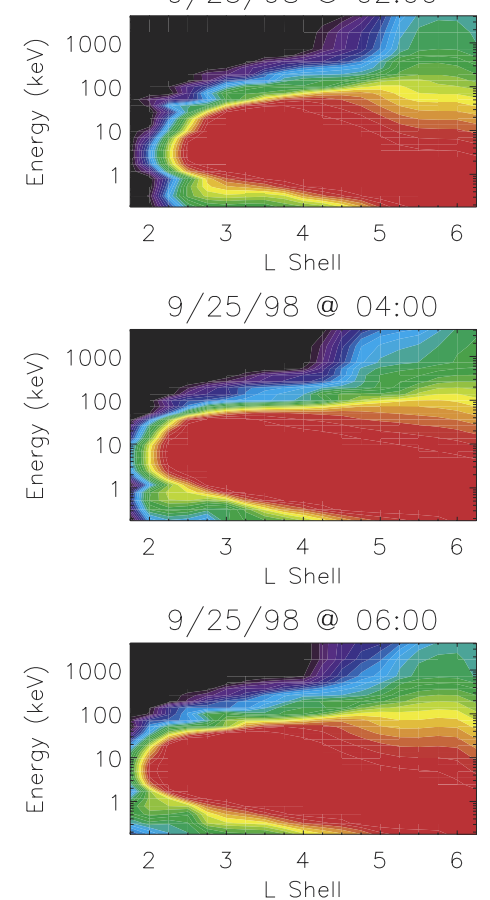

9/25/98@08:00

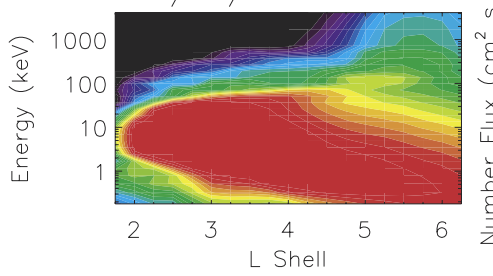

$10^{2}$

$10^{0}$

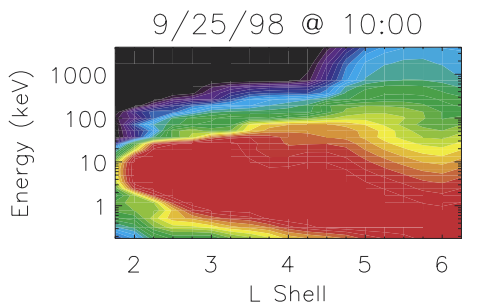

Figure 10. Same spectrogram format as Figure 8, except that all 18 plots are for the September 1998 storm, showing a time progression throughout the entire event (every 2 hours). The plots start at the beginning of the simulation and continue through the early recovery phase of the storm. The MLT location for all of these results is local midnight. 


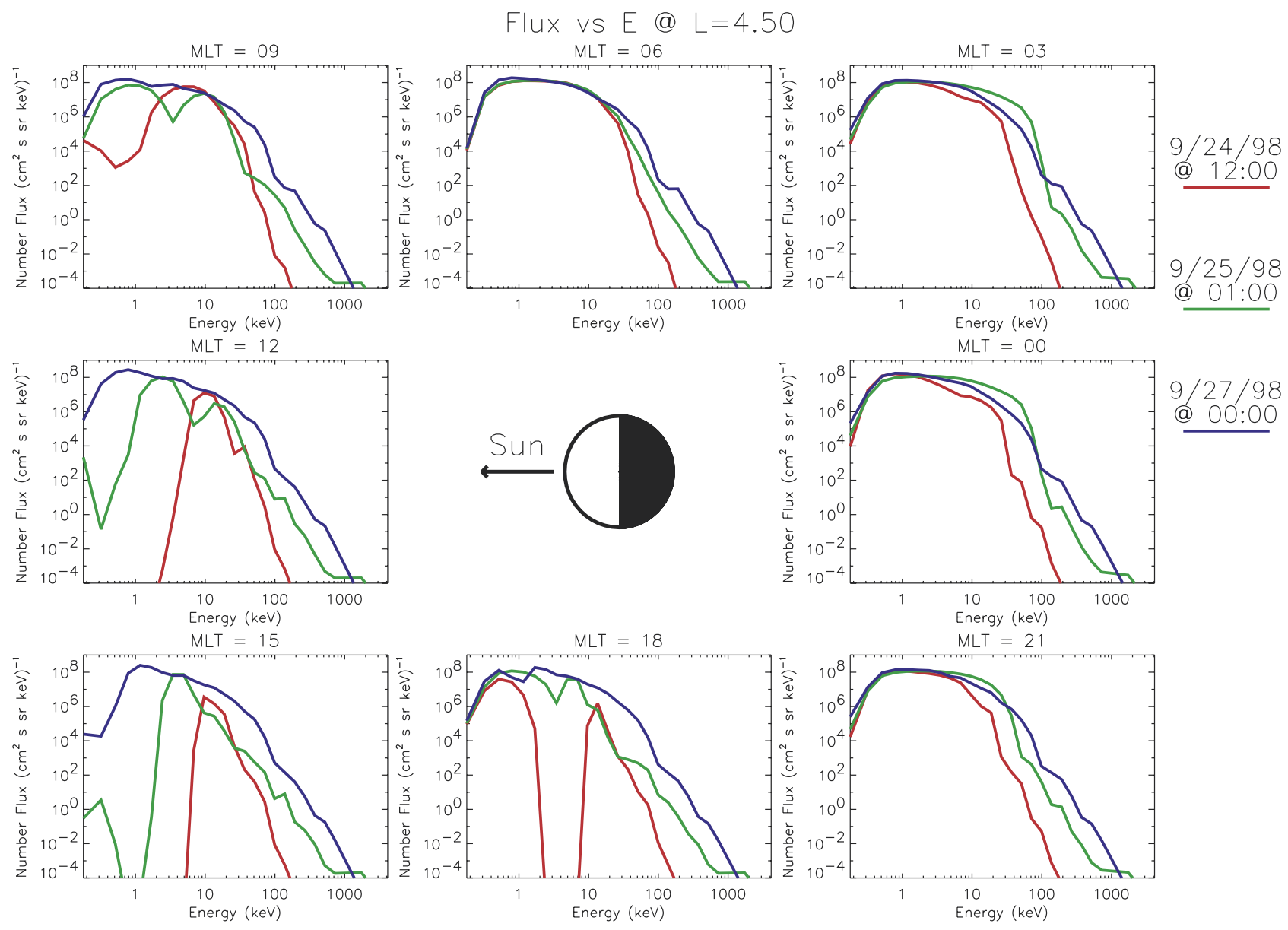

Figure 11. Pitch-angle averaged energy spectra at 8 MLT times around the Earth at L $=4.5$ during the September 1998 storm. The three lines are at three times during the event: (red) prestorm, (green) main phase, and (blue) recovery phase.

The interested reader is referred to that study for more information regarding the energy spectra during this storm.

\section{GEM Campaign Selected Storms Electron Energization}

[20] The storms that occurred on 15 May 1997, 25 September 1998, and 19 October 1998 have been selected for community-wide examination by the National Science Foundation's Geospace Environment Modeling Campaign. The present study examines the formation of relativistic electrons in the inner magnetosphere during these storm events with the radiation belt transport code described above. In this section a different set of boundary conditions and a different electric potential description are used in order to examine the dependence of the energization on potential distribution and boundary condition.

[21] For boundary conditions (that is, particle source terms), the simulations use the observed electron fluxes at geosynchronous orbit $\left(6.6 \mathrm{R}_{\mathrm{E}}\right.$ altitude) from the various LANL geosynchronous spacecraft. Particle data from these satellites are available from the space science group at Los Alamos National Laboratory. In these simulations we use the electron moments (density, perpendicular temperature, and parallel temperature) from the magnetospheric plasma analyzer (MPA) instrument on the spacecraft. These numbers are applied to a bi-kappa distribution for all energies and pitch angles [Thorne and Summers, 1991],

$$
\begin{aligned}
f(E, \mu)= & n \frac{T_{\|}}{T_{\perp}}\left(\frac{m}{2 \pi(\kappa-1.5) T_{\|}}\right)^{3 / 2}\left(\frac{\Gamma(\kappa+1)}{\Gamma(\kappa-.5)}\right) \\
& \cdot\left(1+\frac{E\left(\mu^{2}+\frac{T_{\|}}{T_{\perp}}\left(1-\mu^{2}\right)\right)}{(\kappa-1.5) T_{\|}}\right)^{-\kappa-1} .
\end{aligned}
$$

Like the simulations for the May 1998 storm, kappa is set to 3 . The time cadence of the LANL MPA measurements is $\sim 90 \mathrm{~s}$, offering a rapidly varying boundary condition for the simulations. However, only observations on the nightside are used for the simulation boundary conditions, and therefore gaps of several hours exist. When there is an interval with no LANL satellites on the nightside, then the boundary condition is found by linearly interpolating between the previous and next valid observations. The datasets have also been culled for times when the satellite was on the nightside but was not observing a fresh injection of plasma sheet electrons. That is, the satellites are sometimes in the magnetotail lobes, particularly during 

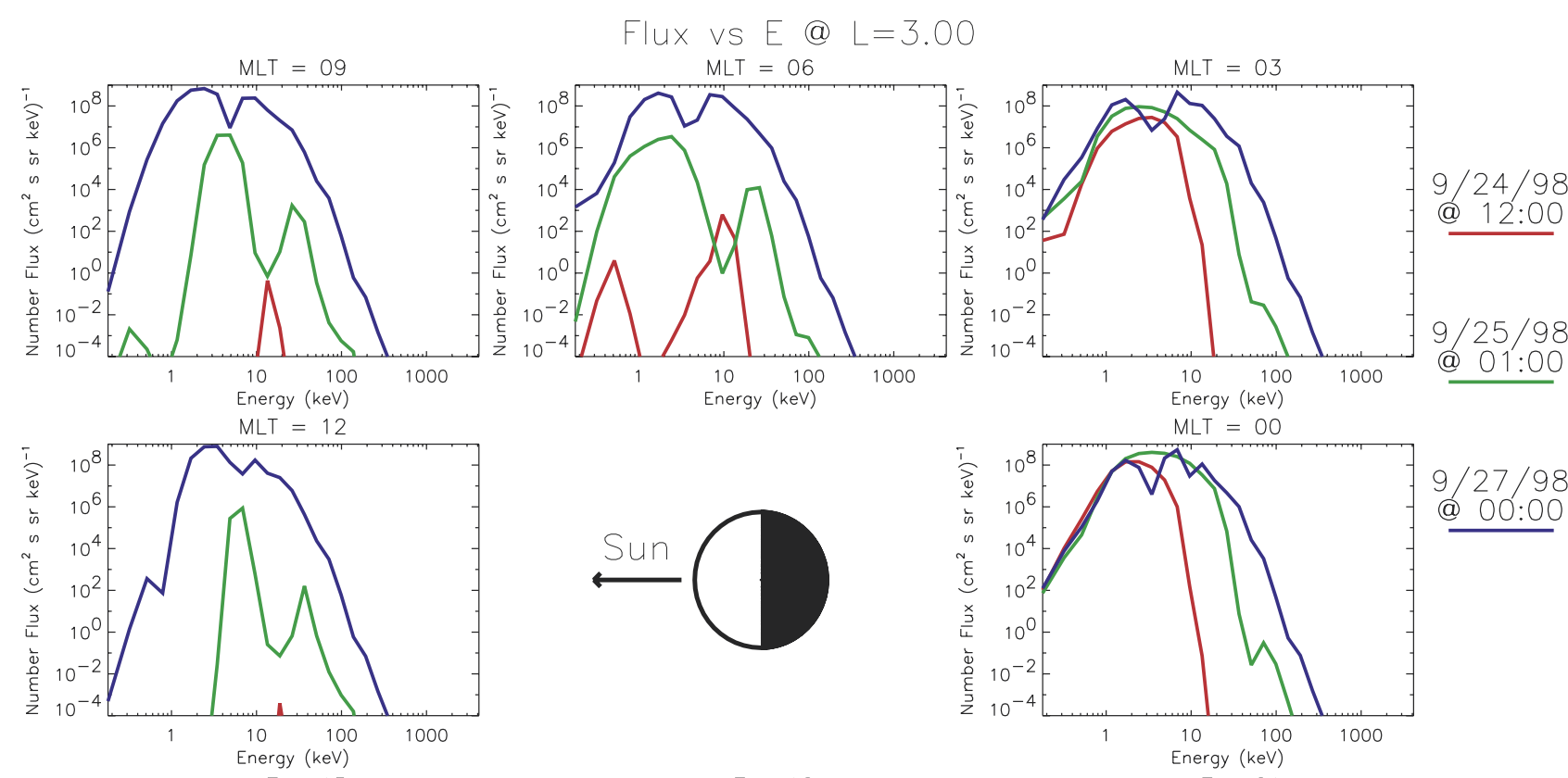

$9 / 25 / 98$

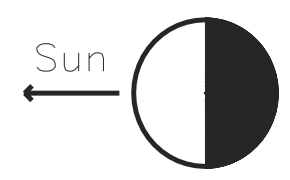

$\mathrm{MLT}=15$
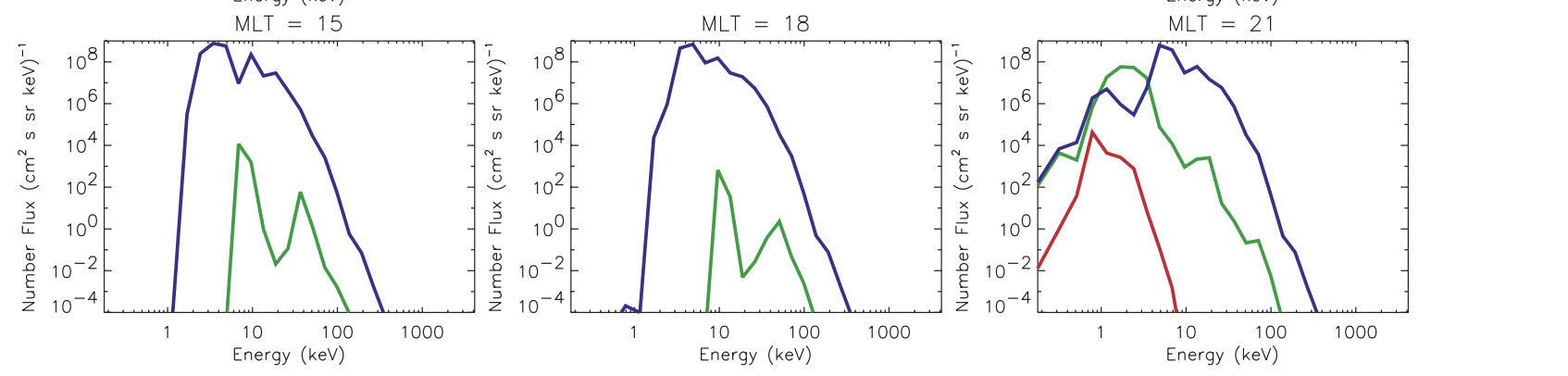

Figure 12. Same as Figure 11 except the results are at $\mathrm{L}=3.00$.

highly stretched field configurations, and thus the measurements are not of the plasma sheet particles which are being injected. When more than one satellite is on the nightside and providing usable data, the observation with the larger density is used as the boundary condition. The boundary condition has no variation in MLT; the fluxes are assumed to be uniform across the nightside. That is, because there are only a few LANL spacecraft operating, assumptions must be made to extend the data to all local times and all universal times. In the present study, spatial variation has been neglected in order to achieve high temporal resolution of the boundary condition electron fluxes. Note that the simulations for the May 1998 storm also used a spatially uniform, high-time-resolution outer boundary condition for the electron fluxes.

[22] These particle fluxes are convected into the simulation domain according to the time-varying electric potential description described by Liemohn et al. [2001]. Here, a twocell convection formula is used that depends on $\mathrm{Kp}$ for the shielding parameter and on the cross polar cap potential (CPCP) for the convection strength. This analytical field model is a combination of the McIlwain [1986] E5D field model but modified for a better description of the stormtime potential variations by changing the activity dependence away from Kp to CPCP. This simple description reproduces the shape of many of the observed drift boundaries of the ring current ions (see Liemohn et al. [2001] for further details). Compared with the AMIE potential patterns, for instance, this model also produces strong convection on the nightside, which is needed to get good agreement with ion data [Khazanov et al., 2004]. In the simulations presented below, the CPCP values are from the AMIE technique. The time cadence of the AMIE CPCP values is $5 \mathrm{~min}$. As found in the previous section, this time cadence provides an adequate level of variability to the electric field to inject the particles deep into the inner magnetosphere. By using a different electric field model, the dependence of this energization on the electric potential description will be assessed.

[23] The CPCP values used in the simulations are shown in Figure 5. The electron densities from the LANL MPA data set used as a boundary condition for these simulations are shown in Figure 6. Gaps in the boundary condition data set appear as straight lines between points. For reference, the observed Dst index values are shown in Figure 7. This index is provided here to show the relative magnitudes of the three storms and the timing of the various phases of the events. Shown in each subplot as black dots along the Dst curve are the times selected for the following figures. In general, the six times are as follows: prestorm, main phase, Dst minimum, and three recovery phase times.

[24] Figure 8 shows energy versus L shell spectrograms at midnight (MLT $=0$ ) at the six times (the six rows) during 


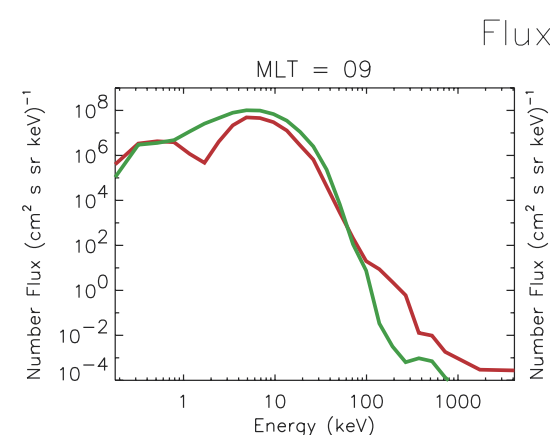

Flux vs E @ L=4.50, 9/25/98@ 06:00
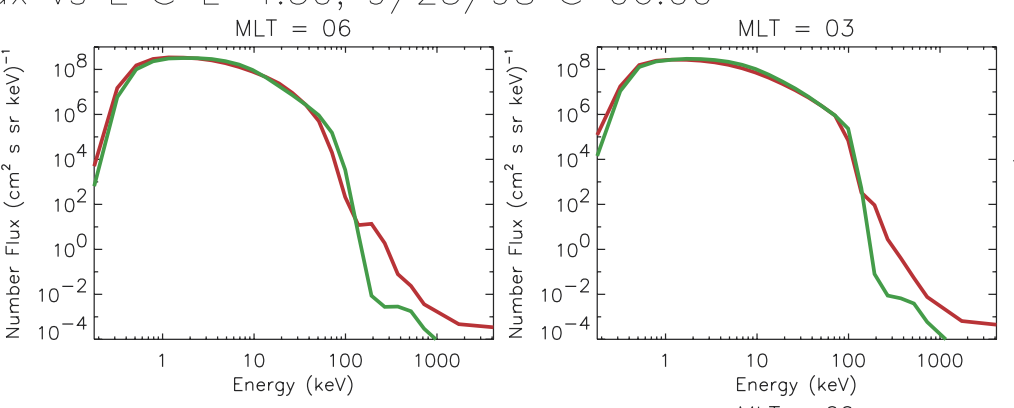

5-minute variance

$\mathrm{MLT}=12$
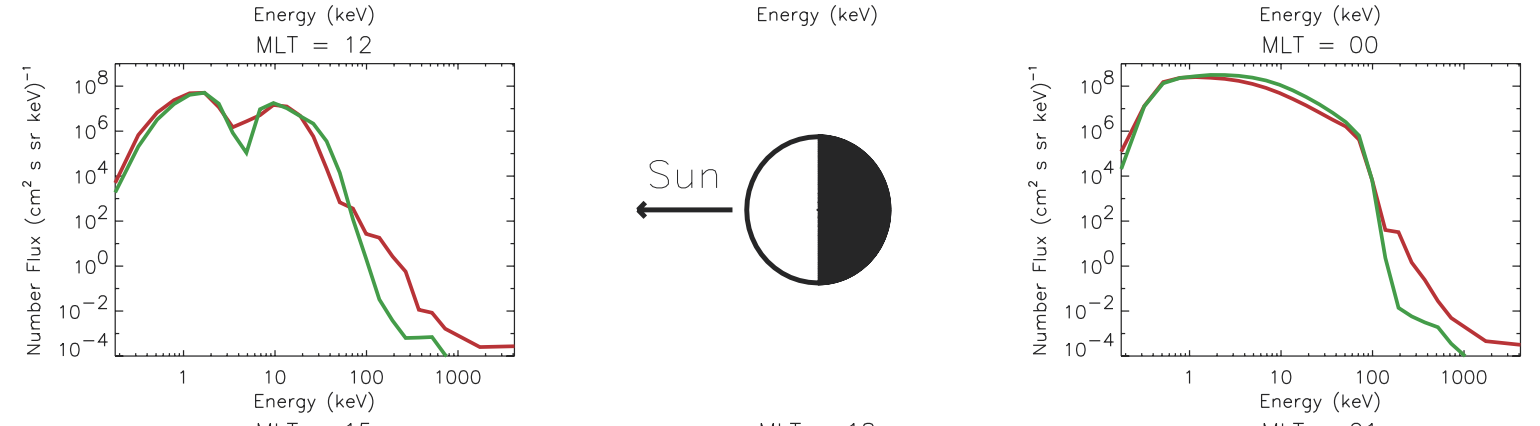

3-hour variance
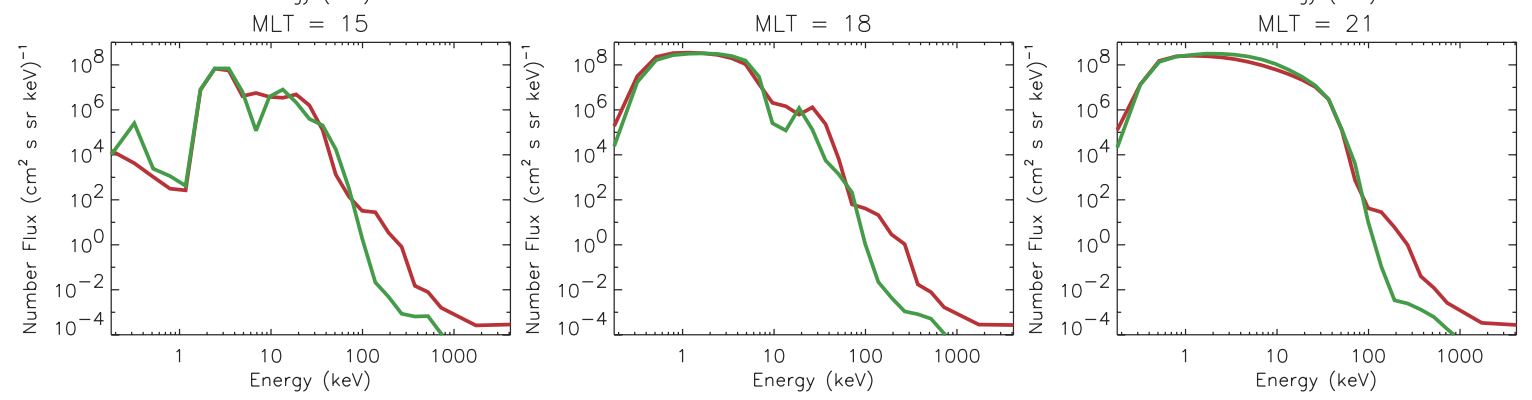

Figure 13. The same energy spectra format as Figure 11, except that the two lines in each plot are for the two time-cadence options run for the September 1998 storm. The results are for $\mathrm{L}=4.5$ near the peak of the storm.

each event (the three columns). The color scale has been chosen to highlight the high-energy end of the spectrum, and so the low-energy peak of the electron fluxes is often appear saturated. The injection of the electrons into the inner magnetosphere is seen to progress throughout each event, with a dramatic increase in injection during the main phase of the storm. Figure 9 is a similar plot but for noon $(\mathrm{MLT}=12)$. In comparing Figures 8 and 9, it is seen that the electrons enter on the nightside and drift eastward toward the dayside, where the enhancement occurs at a later time. As convection enhances during the storm, these particles are injected deeper into the inner magnetosphere (that is, closer to the Earth) and are adiabatically energized in the process. This energization is clearly seen in the results. Because the eastward gradient-curvature drift of the electrons is energydependent, the higher-energy electrons do not penetrate as far in toward the Earth as the lower-energy particles. This is illustrated as the creation of a "nose" in the spectrograms. Because of the continuously fluctuating electric field, the electrons are also randomly diffused in radial distance. This results in a net acceleration of the electrons (because the source term is at large $\mathrm{L}$ values). This process is especially noticeable in the high-energy tail of the distribution. By comparing the prestorm and poststorm figures, it is seen that many electrons have been injected into the inner magneto- sphere and a significant seed population for the radiation belts has been formed.

[25] Figure 10 is a similar plot format except that it shows results for the September 1998 event only, plotted every 2 hours of simulation time at MLT $=00$ from the start of the run up through the minimum in Dst. This plot shows the time evolution of the distribution in the inner magnetosphere and clearly shows that the injection timescales are much faster than the diffusion timescales. That is, most of the injection is due to convective forces pushing the electrons into the inner magnetosphere.

[26] To quantify the magnitude of this increase, Figures 11 and 12 show line plots of the pitch-angle averaged energy spectra at two L values (4.5 and 3.0 for Figures 11 and 12, respectively) at three times during the September 1998 event (prestorm, main phase, and late recovery; the red, green, and blue lines, respectively). The 8 line plots are shown every 3 hours of MLT in their respective positions around the Earth (drawn in the center of each figure). The increases in flux are dramatic, with many orders of magnitude more electrons in the simulation domain than before the magnetic storms. The results at $\mathrm{L}=3.0$ show this increase especially well. It is interesting to note how bumpy the energy spectra appear in Figures 11 and 12. This is due to the time variability of both the boundary conditions and the electric field strength, as 

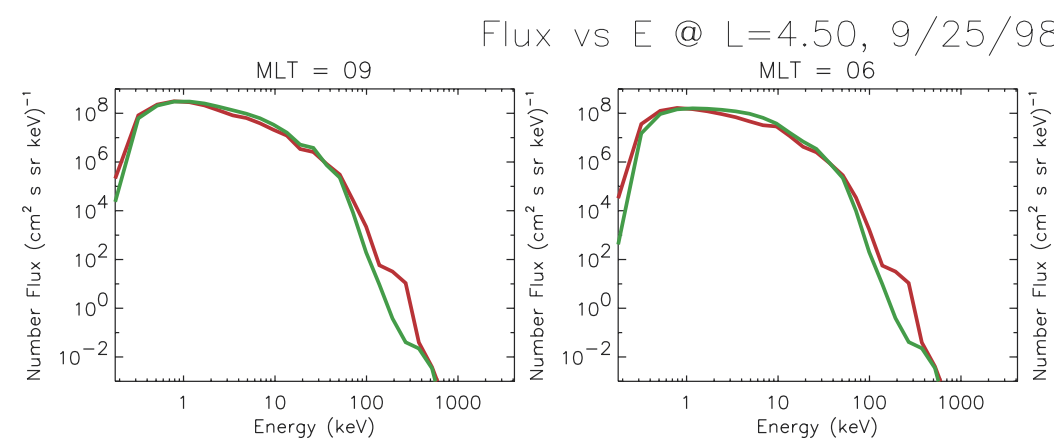

@ 22:00
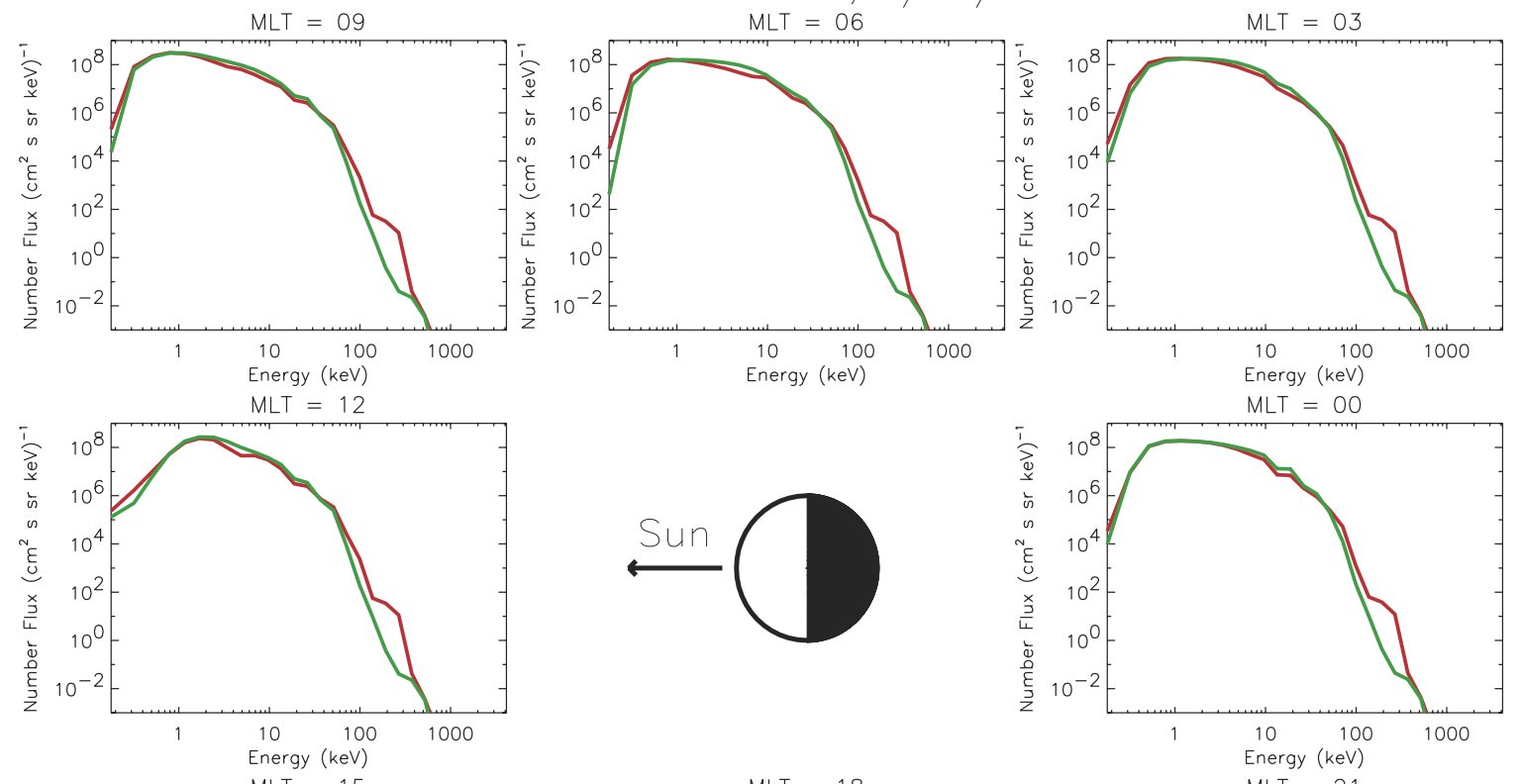

5-minute variance

3-hour variance

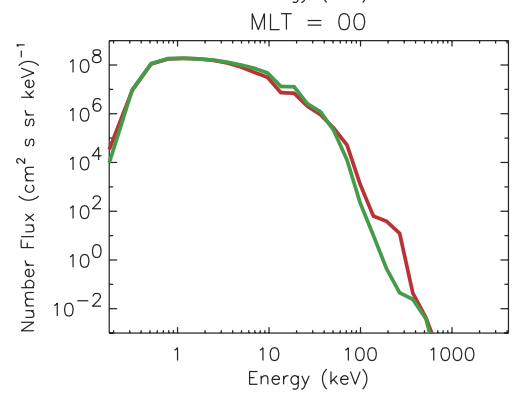

$M L T=15$
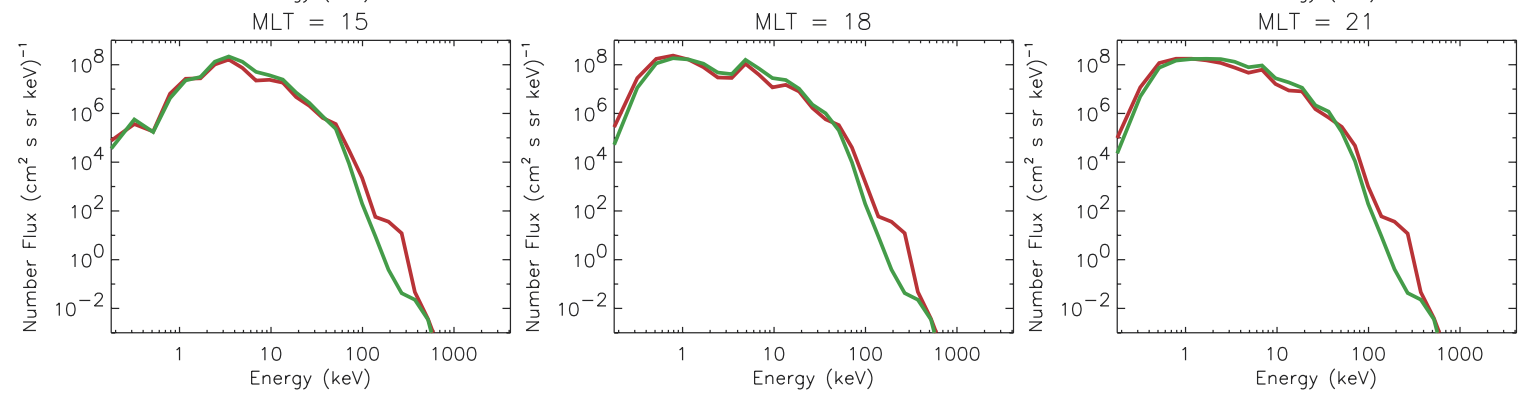

Figure 14. Same as Figure 13 except that the chosen time is in the recovery phase of the storm.

seen in Figures 5 and 6 . The convolution of these parameters (along with the variance in $\mathrm{T}_{\|}$and $\mathrm{T}_{\perp}$ ) leads to a complicated structure in the electron distribution function in the inner magnetosphere. Certain particles will be in drift resonance with the fluctuations in the electric field and will be injected faster and deeper than the electrons at other energies. Conversely, an interval of low density at the nightside boundary accompanied by high convection will result in a net loss of electrons from the inner magnetosphere (flow out the dayside boundary).

[27] It is interesting to note that the three events have very different Dst minima and time sequences and also quite different peak and integrated CPCP values. However, all three events resulted in substantially enhanced electrons in the inner magnetosphere. This is because the electric field was allowed to fluctuate with a fast cadence, and the particles were able to convect and diffuse in toward the Earth throughout the event.

[28] To investigate this dependence, the September 1998 simulation was rerun with a different set of boundary conditions. Specifically, the CPCP and LANL electron moments were averaged over 3 hour bins. As in section 3, this choice of an averaging timescale is to approximate a Kp-driven simulation, which has a 3-hour time cadence. The CPCP and electron boundary conditions still change every time step in the new simulation, but the values are now interpolated from these 3-hour averages, rather than from the high time resolution input files. A comparison of the results from the two simulations is given in Figures 13 and 14. These two plots are similar to Figures 11 and 12, except the two lines drawn in each subplot are for the two simulations and all results are shown for main (Figure 13) and recovery (Figure 14) phases of the storm. It is seen that the fluxes are up to three orders of magnitude higher in the high-time-cadence simulation results, especially at the highenergy $(10-1000 \mathrm{keV})$ end of the spectrum. This is due to the fluctuations in the electric field resonating with the drift periods of these electrons, preferentially accelerating them via a net inward radial drift. This enhancement is seen clearly in the $100 \mathrm{~s}$ of $\mathrm{keV}$ energy range at this radial distance $(L=4.5)$, which is exactly where a seed population is needed for the production of outer zone radiation belt relativistic electrons.

[29] The point of this numerical experiment is to show that a simulation of a real event with measured particle inputs must have a high time resolution E-field update in order to produce a seed population for the radiation belts, which are often observed to increase in the days following a magnetic storm. It should be pointed out, however, that the flux differences vary from one storm to another and are a function of not only the intensity of the particular magnetic storm but also the previous history of Dst development. A comparison of the results presented in this section and the previous section imply that the energization depends more on the variability of the inner magnetospheric electric fields than on the specific choice of the electric potential descrip- 
tion employed in the simulation. Large particle flux increases in the $10-1000 \mathrm{keV}$ range were generated with both electric field models (the AMIE potentials and the McIlwain potentials), as long as the time cadence of the variation was 5 min or less.

\section{Conclusions}

[30] Four storms were examined to quantify the influence of electric field fluctuations on the development of the electron distribution function in the inner magnetosphere. It was found that the global electric field pattern should be updated with a cadence of 5 min or less in order to properly simulate the enhancement of the 100-500 keV electrons in the $L=3-5$ range. This is the energy range and spatial region for the seed population of the $\mathrm{MeV}$ electrons in the outer zone radiation belt. This study showed that E-field fluctuations are critical to the formation of this seed population during magnetic storms. These electrons have drift periods close to the fluctuation period of the global potential pattern, and therefore they experience energization. A model that does not take into account these rapid fluctuations of the inner magnetospheric electric field will underestimate the fluxes of these electrons. Differences were particularly large (up to several orders of magnitude) relative to simulation results with a 3 -hour time cadence, analogous to a Kp-driven electric field model.

[31] This study shows that by varying the global structure of the electric field at a high time cadence, specifically $5 \mathrm{~min}$ or less between potential pattern updates, a stochastic motion of the particle drift paths occurs. These random changes result in radial diffusion, and because the particle source is at the outer simulation boundary, the net effect is an inward propagation of particles and thus an energization. As was stressed in section 2, we did not employ the classic radial diffusion process [Schulz and Lanzerotti, 1974] in equation (1). These diffusion coefficients are small and cannot explain the rapid rise in the electron flux [e.g., Chen et al., 1993, 1994; Sheldon, 1994; Beutier and Boscher, 1995; Bourdarie et al., 1996, 1997]. Instead, we took into account electric field fluctuations on a timescale comaparable with or less than the drift period of radiation belt electrons from the realistic potential distributions provided by the AMIE model. Radial diffusion by nature is a stochastic process that is driven by electric and magnetic field fluctuations that are included in the model through electric and magnetic drifts. This study simply shows that stochastic transport and energization can be obtained if the simulation includes a rapidly fluctuating, realistic electric field description. It also quantifies the rapidness with which it much change in order to accurately replicate this diffusion process.

[32] Acknowledgments. Funding in support of this study was partially provided by NASA grants NAG5-5030, NAG5-10297 and NAG10850 and by NSF grant ATM-0090165. We would like to thank Michelle Thomsen for providing the LANL particle data used as input to the GEMevent storm simulations in this study.

[33] Shadia Rifai Habbal thanks Michel Blanc and another referee for their assistance in evaluating this paper.

\section{References}

Albert, J. M. (2001), Comparison of pitch angle diffusion by turbulent and monochromatic whistler waves, J. Geophys. Res., 106, 8477.
Anderson, P. C., D. L. Carpenter, K. Tsuruda, T. Mukai, and F. J. Rich (2001), Multisatellite observations of rapid subauroral ion drifts (SAID), J. Geophys. Res., 106, 29,585.

Beutier, T., and D. Boscher (1995), A three-dimensional analysis of the electron radiation belt by the Salammbô, J. Geophys. Res., 100, 14,853. Boonsiriseth, A., R. M. Thorne, G. Lu, V. K. Jordanova, M. F. Thomsen, D. M. Ober, and A. J. Ridley (2001), A semiempirical equatorial mapping of AMIE convection electric potentials (MACEP) for the January 10, 1997, magnetic storm, J. Geophys. Res., 106, 12,903.

Borovsky, J. E., M. F. Thomsen, and R. C. Elphic (1998), The driving of the plasma sheet by the solar wind, J. Geophys. Res., 103, 17,617.

Bourdarie, S., D. Boscher, T. Beutier, J.-A. Sauvaud, and M. Blanc (1996), Magnetic storm modeling in the Earth's electron belt by the Salammbô code, J. Geophys. Res., 101, 27,171.

Bourdarie, S., D. Boscher, T. Beutier, J.-A. Sauvaud, and M. Blanc (1997), Electron and proton radiation belt dynamic simulations during storm periods: A new asymmetric convection-diffusion model, J. Geophys. Res., 102, 17,541.

Brice, N. M. (1967), Bulk motion of the magnetosphere, J. Geophys. Res., $72,1246$.

Burch, J. L., et al. (2001), Views of Earth's magnetosphere with the IMAGE satellite, Science, 291, 619

Burke, W. J., N. C. Maynard, M. P. Hagan, R. A. Wolf, G. R. Wilson, L. C. Gentile, M. S. Gussenhoven, C. Y. Huang, T. W. Garner, and F. J. Rich (1998), Electrodynamics of the inner magnetosphere observed in the dusk sector by CRRES and DMSP during the magnetic storm of June 4-6, 1991, J. Geophys. Res., 103, 29,399.

Chen, M. W., M. Schulz, L. R. Lyons, and D. J. Gorney (1993), Stormtime transport of ring current and radiation belt ions, J. Geophys. Res., 98, 3835 .

Chen, M. W., L. R. Lyons, and M. Schulz (1994), Simulations of phase space distributions of storm-time proton ring current, J. Geophys. Res., 99, 5745.

Chen, M. W., M. Schulz, G. Lu, and L. R. Lyons (2003), Quasi-steady drift paths in a model magnetosphere with AMIE electric field: Implications for ring current formation, J. Geophys. Res., 108(A5), 1180, doi:10.1029/ 2002JA009584.

Ebihara, Y., and M. Ejiri (2000), Simulation study on fundamental properties of the storm-time ring current, J. Geophys. Res., 105, 15,843.

Ejiri, M. (1978), Trajectory traces of charged particles in the magnetosphere, J. Geophys. Res., 83, 4798.

Farrugia, C. J., et al. (2002), Wind and ACE observations during the great flow of 1-4 May 1998: Relation to solar activity and implications for the magnetosphere, J. Geophys. Res., 107(A9), 1240, doi:10.1029/ 2001JA000188.

Fejer, B. G., et al. (1990), Low and mid-latitude ionospheric electric fields during the January 1984 GISMOS campaign, J. Geophys. Res., 95, 2367.

Fok, M.-C., T. E. Moore, and W. N. Spjeldvik (2001), Rapid enhancement of radiation belt electron fluxes due to substorm dipolarization of the geomagnetic field, J. Geophys. Res., 106, 3873.

Foster, J. C., and H. B. Vo (2002), Average characteristics and activity dependence of the subauroral polarization stream, J. Geophys. Res., 107(A12), 1475, doi:10.1029/2002JA009409.

Foster, J. C., J. M. Holt, R. G. Musgrove, and D. S. Evans (1986), Ionospheric convection associated with discrete levels of particle precipitation, Geophys. Res. Lett., 13, 656.

Gloeckler, G., et al. (1999), Unusual composition of the solar wind in the 2-3 May 1998 CME observed with SWICS on ACE, Geophys. Res. Lett., 26, 157.

Goldstein, J., B. R. Sandel, W. T. Forrester, and P. H. Reiff (2003), IMFdriven plasmasphere erosion of 10 July 2000, Geophys. Res. Lett., 30(3), 1146, doi:10.1029/2002GL016478.

Hudson, M. K., S. R. Elkington, J. G. Lyon, V. A. Marchenko, I. Roth, M. Temerin, and M. S. Gussenhoven (1996), MHD/particle simulations of radiation belt formation during a storm sudden commencement, in Radiation Belts: Models and Standards, Geophys. Monogr. Ser, vol. 97, edited by J. F. Lemaire, D. Heynderickx, and D. N. Baker, p. 57, AGU, Washington, D. C.

Jordanova, V. K., L. M. Kistler, J. U. Kozyra, G. V. Khazanov, and A. F. Nagy (1996), Collisional losses of ring current ions, J. Geophys. Res., $101,111$.

Jordanova, V. K., J. U. Kozyra, A. F. Nagy, and G. V. Khazanov (1997), Kinetic model of the ring current-atmosphere interactions, J. Geophys. Res., 102, 14,279.

Jordanova, V. K., L. M. Kistler, C. J. Farrugia, and R. B. Torbert (2001), Effects of inner magnetospheric convection on ring current dynamics: March 10-12, 1998, J. Geophys. Res., 106, 29,705.

Khazanov, G. V., M. W. Liemohn, J. U. Kozyra, and T. E. Moore (1998), Global superthermal electron transport: Photoelectron and plasma sheet electron sources, J. Geophys. Res., 103, 23,485. 
Khazanov, G. V., M. W. Liemohn, E. N. Krivorutsky, J. M. Albert, J. U. Kozyra, and B. E. Gilchrist (1999), Relativistic electron beam propagation in the Earth's magnetosphere, J. Geophys. Res., 104, 28,587.

Khazanov, G. V., K. V. Gamayunov, V. K. Jordanova, and E. N. Krivorutsky (2002), A self-consistent model of the interacting ring current ions and electromagnetic ion cyclotron waves, initial results: Waves and precipitating fluxes, J. Geophys. Res., 107(A6), 1085, doi:10.1029/ 2001JA000180.

Khazanov, G. V., M. W. Liemohn, T. S. Newman, M.-C. Fok, and A. J. Ridley (2004), Magnetospheric convection electric field dynamics and stormtime particle energization: Case study of the magnetic storm of 4 May 1998, Ann Geophys., 22, 497.

Li, X., I. Roth, M. Temerin, J. R. Wygant, M. K. Hudson, and J. B. Blake (1993), Simulation of the prompt energization and transport of radiation belt particles during the March 24, 1991 SSC, Geophys. Res. Lett., 20, 2433.

Liemohn, M. W., J. U. Kozyra, M. F. Thomsen, J. L. Roeder, G. Lu, J. E. Borovsky, and T. E. Cayton (2001), Dominant role of the asymmetric ring current in producing the stormtime Dst*, J. Geophys. Res., 106, 10,883.

Lyons, L. R., R. M. Thorne, and C. F. Kennel (1972), Pitch-angle diffusion of radiation belt electrons with the plasmasphere, J. Geophys. Res., 77, 3455

Ma, C.-Y., and D. Summers (1999), Correction to "Formation of power-law energy spectra in space plasmas by stochastic acceleration due to whistlermode waves," Geophys. Res. Lett., 26, 1121

McIlwain, C. E. (1974), Substorm injection boundaries, in Magnetospheric Physics, edited by B. M. McCormac, p. 143, D. Reidel, Norwell, Mass

McIlwain, C. E. (1986), A Kp dependent equatorial electric field model, Adv. Space Res., 6(3), 187.

Nishida, A. (1966), Formation of a plasmapause, or magnetospheric plasma knee by combined action of magnetospheric convection and plasma escape from the tail, J. Geophys. Res., 71, 5669.

Richmond, A. D., and Y. Kamide (1988), Mapping electrodynamic features of the high-latitude ionosphere from localized observations: Technique, J. Geophys. Res., 93, 5741.

Ridley, A. J., G. Lu, C. R. Clauer, and V. O. Papitashvili (1998), A statistical study of the ionospheric convection response to changing interplanetary magnetic field conditions using the assimilative mapping of ionospheric electrodynamics technique, J. Geophys. Res., 103, 4023

Ridley, A. J., G. Lu, C. R. Clauer, and V. O. Papitashvili (1999), Reply, J. Geophys. Res., 104, 4393.
Rowland, D., and J. R. Wygant (1998), The dependence of the large scale electric field in the inner magnetosphere on magnetic activity, J. Geophys. Res., 103, 14,959.

Schulz, M., and L. J. Lanzerotti (1974), Particle Diffusion in the Radiation Belts, Springer-Verlag, New York.

Sheldon, R. B. (1994), Ion transport and loss in the Earth's quiet ring current: 2. Diffusion and magnetosphere-ionosphere coupling, J. Geophys. Res., 99, 5705.

Skoug, R. M., et al. (1999), A prolonged $\mathrm{He}^{+}$enhancement within a coronal mass ejection in the solar wind, Geophys. Res. Lett., 26, 161.

Stern, D. P. (1975), The motion of a proton in the equatorial magnetosphere, J. Geophys. Res., 80, 595.

Summers, D., and C.-Y. Ma (2000), Rapid acceleration of electrons in the magnetosphere by fast-mode MHD waves, J. Geophys. Res., 105, 15,887. Thorne, R. M., and D. Summers (1991), Enhancement of wave growth for warm plasmas with a high-energy tail distribution, J. Geophys. Res., 96 , 217

Volland, H. (1973), A semiempirical model of large-scale magnetospheric electric fields, J. Geophys. Res., 78, 171.

Weimer, D. R. (1996), A flexible, IMF dependent model of high-latitude electric potentials having "space weather" applications, Geophys. Res. Lett., 23, 2549.

Wygant, J., D. Rowland, H. J. Singer, M. Temerin, F. Mozer, and M. K. Hudson (1998), Experimental evidence on the role of the large spatial scale electric field in creating the ring current, J. Geophys. Res., 103, $29,527$.

Yeh, H.-C., J. C. Foster, F. J. Rich, and W. Swider (1991), Storm time electric field penetration observed at mid-latitude, J. Geophys. Res., 96, 5707.

M.-C. Fok, Laboratory for Extraterrestrial Physics, Code 692, NASA Goddard Space Flight Center, Greenbelt, MD 20771, USA. (meiching.fok@gsfc.nasa.gov)

G. V. Khazanov, National Space Science and Technology Center, NASA Marshall Space Flight Center, Huntsville, AL 35899, USA. (george. khazanov@msfc.nasa.gov)

M. W. Liemohn and A. J. Ridley, Space Physics Research Laboratory, University of Michigan, Ann Arbor, MI 48109, USA. (liemohn@umich. edu; ridley@umich.edu)

T. S. Newman, Computer Science Department, University of Alabama in Huntsville, Huntsville, AL 35899, USA. (tnewman@cs.uah.edu) 